McGill-93/14

UdeM-LPN-TH-93-154

OCIP/C-93-7

hepph-9307223

June 1993

\title{
BOUNDING ANOMALOUS GAUGE-BOSON COUPLINGS
}

\author{
C.P. Burgess ${ }^{a}$ Stephen Godfrey, ${ }^{b}$ Heinz König, ${ }^{b}$ David London ${ }^{c}$ and Ivan Maksymyk ${ }^{c}$ \\ ${ }^{a}$ Physics Department, McGill University \\ 3600 University St., Montréal, Québec, CANADA, H3A $2 T 8$. \\ ${ }^{b}$ Ottawa-Carleton Institute for Physics \\ Physics Department, Carleton University \\ Ottawa, Ontario, CANADA, K1S 5B6. \\ ${ }^{c}$ Laboratoire de Physique Nucléaire, l'Université de Montréal \\ C.P. 6128, Montréal, Québec, CANADA, H3C 3J\%.
}

\begin{abstract}
We investigate indirect phenomenological bounds on anomalous three-gauge-boson couplings. We do so by systematically determining their one-loop implications for precision electroweak experiments. We find that these loop-induced effects cannot be parametrized purely in terms of the parameters $S, T$ and $U$. Like some other authors, we find many cancellations among the loop-induced effects, and we show how to cast the low-energy effective theory into a form which makes these cancellations manifest at the outset. In a simultaneous fit of all CP conserving anomalous three-gauge-boson couplings, our analysis finds only weak phenomenological constraints.
\end{abstract}




\section{Introduction}

The experimental couplings of the electroweak gauge bosons to light fermions have now been quite well explored, particularly using low-energy lepton scattering experiments and precision measurements at the $Z$ resonance. However, accurate experimental information is not available for the self-interactions among the gauge bosons. This situation is likely to be partially alleviated once the centre-of-mass energies at the LEP $e^{+} e^{-}$collider are raised above the threshold for $W^{ \pm}$pair production (LEP200). Given a sufficiently large sample of $W^{ \pm}$pairs, direct information becomes available concerning the nature of the $W W \gamma$ and $W W Z$ couplings. It is expected that a deviation (of $10 \%$ or more) of the three gauge-boson vertices (TGV's) from their standard model (SM) values can be detected in this way [1], [2], [3].

The key question is whether there are any kinds of new, nonstandard physics that can give rise to this large a deviation from SM predictions for the TGV's, and yet which might not have been detected elsewhere in other low-energy experiments. As might have been expected, a great deal of effort has been expended on researching this subject, leading to a dauntingly large literature on the subject [4].

Two approaches to answering this question may be taken, depending on one's theoretical prejudices.

- Theoretically-Motivated Bounds: The first approach is to use (sometimes fairly benign) assumptions about the nature of the new physics in order to obtain an estimate of how large the anomalous TGV's might be. The strength of this type of conclusion is then inversely related to the restrictiveness of the assumptions that are required in order to derive it. The thrust of this line of thinking is usually to (reasonably convincingly) argue that induced anomalous TGV's are unlikely to be larger than $\mathrm{O}(1 \%)$ of their SM counterparts. If true, this would make their detection at LEP improbable.

There are two broad classes of new physics for which this conclusion is probably true. Firstly, if the new physics is perturbatively coupled to the electroweak gauge bosons, then its contributions to TGV's are of order $(g / 4 \pi)^{2} \sim 10^{-3}$, where $g$ is an electroweak coupling constant. Since the transverse gauge bosons couple with a universal strength, this estimate includes a great many models. Any couplings between the new physics and the longitudinal gauge bosons need not be so small, however, and so a strongly-coupled symmetry-breaking sector might be considered.

In this case, a second line of reasoning leads to a similar conclusion concerning the detectablity of anomalous TGV's [5]. To the extent that the low-energy $W^{ \pm}$physics is dominated purely by the couplings of the longitudinal modes, it may be parametrized 
using familiar techniques of chiral perturbation theory [6]. It is therefore quite plausible that the size of these effective interactions depends on the weak scale and the unknown scale, $M$, of the new physics in the same way as do the corresponding couplings in the lowenergy chiral limit of QCD. This dependence may be succinctly summarized by the rules of 'Naive Dimensional Analysis' [7], which indicate that the relative size of anomalous and SM TGV's should be $O\left(M_{W}^{2} / M^{2}\right)$. Again, for $M$ as large as a few TeV, as might be expected for a strongly-interacting Higgs sector for example, we are led to expect anomalous TGV's of $O(1 \%)$. In either case, these are too small to be observed.

A complementary line of argument is based on naturalness [8]. One way of phrasing this argument states that anomalous TGV's should be of the same size as other new-physics contributions to the purely gauge sector (e.g. 'oblique' corrections) [9], [10], [11], since there are no symmetries that could naturally enforce a relative size difference. Since these oblique corrections are bounded by precision electroweak measurements to be $\lesssim O(1 \%)$, so the argument goes, anomalous TGV's can also be expected to be, at most, this large.

- Purely Phenomenological Bounds: The second, more conservative tack that may be taken in determining the potential size of anomalous TGV's is to put theoretical prejudices aside and ask for purely phenomenological bounds. In this case we ask whether anomalous couplings that are large enough to be detected can be already excluded based on other low-energy measurements. The potential bounds of this sort arise either due to the direct probing of nonstandard gauge-boson couplings in hadron colliders [3], [12], [13], [14], or from their indirect influence on precisely measured quantities, through loops. Although there is agreement that existing hadron machines cannot rule out detectable nonstandard TGV's at LEP200 [13], the extraction of bounds from loops has been more controversial $[15]$.

Here again the most recent analyses may be classified according to the assumptions that are made. Some have entailed a weakly-coupled framework within which the standardmodel gauge group is linearly realized at low energies by including an explicit light physical Higgs particle [8], [16], [17]. In all of these studies it is found that detectably large anomalous TGV's cannot be ruled out purely by phenomenology. Other workers [18], [19] have instead not assumed the existence of a light Higgs, in an effort to extract bounds that are less constrained by assumptions concerning how the gauge symmetry is realized. Again the detectable TGV's are not ruled out on purely phenomenological grounds.

It is natural to ask why purely phenomenological bounds should be pursued at all, given that reasonably persuasive theoretical arguments indicate that detectable anomalous TGV's are unlikely. Our own point of view is that neither a purely theoretical estimate, nor a purely phenomenological analysis is sufficient in itself. We can only hope to learn 
anything if both types of investigations are performed, since it is only through the comparison of both, and their subsequent confrontation with the direct measurements at LEP, that we learn something about the nature of any new physics.

The present paper is intended as a contribution to the purely phenomenological line of thought. We wish here to determine the constraints on CP-preserving anomalous TGV's, with an absolute minimum of assumptions about the nature of the new physics from which they are generated. As with the previous analyses of refs. [8], [17], [18] and [19], we assume that the scale, $M$, that is associated with the new physics is high enough in comparison with the weak scale, $M_{W}$, to justify an effective-lagrangian treatment that is controlled by powers of $1 / M$. Integrating out the physics at scale $M$ generates a host of effective interactions, including anomalous TGV's among others:

$$
\mathcal{L}_{\text {eff }}\left(\mu^{2}=M^{2}\right)=\mathcal{L}_{S M}+\mathcal{L}_{T G V}+\mathcal{L}_{\text {rest }} .
$$

Here $\mathcal{L}_{S M}$ is the SM lagrangian, $\mathcal{L}_{T G V}$ represents the anomalous TGV interactions and $\mathcal{L}_{\text {rest }}$ denotes all of the other effective operators. Only some of the effective interactions in $\mathcal{L}_{\text {rest }}$ contribute at tree level to well-measured low-energy observables, and so only these are presently well-constrained by the data. All other operators — including TGV's in particular - do not contribute in this way and so are only bounded to the extent that they generate the better-constrained operators as the effective theory is run down from $\mu=M$ to the much lower scales where the low-energy measurements are ultimately performed. Our purpose here is to compute which operators are generated by TGV's in this way, and so to indirectly bound their coefficients.

Before describing our conclusions, it is useful to orient our calculation in relation to the others that have recently been performed. Our calculation differs from those of refs. [8], [16] and [17] in that we assume that the dominant degrees of freedom that govern the loop contribution of TGV's to low-energy observables are only the presently known particles (including the top quark). In particular, we do not assume a light Higgs boson and do not linearly realize the electroweak gauge group. As was emphasized in ref. [15], one can choose to realize this gauge symmetry nonlinearly, or to ignore it completely (apart from its $U_{\mathrm{em}}(1)$-invariant subgroup), and in both cases one is led to precisely the same lowenergy effective lagrangian [20]. The price to be paid is that the new-physics scale cannot be arbitrarily large: $M_{W} / M \gtrsim g / 4 \pi$, or else perturbative unitarity is lost.

Our analysis also differs in important ways from those of refs. [18] and [19]. Perhaps the most basic difference lies in the number of effective operators that are considered. We use the most general effective interactions of ref. [1] that are consistent with $\mathrm{CP}$ conservation. 
Our calculations therefore include the five effective couplings, $\Delta \kappa_{\gamma}, \Delta \kappa_{z}, \Delta g_{1 z}, \lambda_{z}$, and $\lambda_{\gamma}$ (see the following section for detailed definitions). Our results may be compared to those of ref. [19] by taking $\Delta g_{1 z}=0$, and to those of ref. [18] by choosing $\lambda_{z}=\lambda_{\gamma}=0$. It should be pointed out that the neglect of $\lambda_{z}$ and $\lambda_{\gamma}$ in ref. [18] is what would be expected if the new physics were to produce an effective theory that satisfies the power-counting rules [7] that have been found from experience with chiral perturbation theory in QCD. We do not make this assumption here, however, since it is not a generic feature of all underlying theories at scale $M .^{1}$

A further difference with other workers arises because the authors of refs. [8] and [18] also make explicit uses of quadratic divergences in arriving at their bounds. In the present language, their calculation corresponds to an estimate of the size of direct contributions of new physics to $\mathcal{L}_{\text {rest }}$ at scale $\mu=M$, rather than of the induced effects at low energies due to the TGV's defined at this scale [15]. This distinction is less important in the linearly-realized case, where the low-energy divergences are less severe.

A calculation of the purely low-energy loop effects of TGV's has recently been made without the assumption of a light Higgs in ref. [19]. These authors use the formalism of oblique corrections, as parametrized by Peskin and Takeuchi's $S, T$ and $U$ [9], to obtain their low-energy bounds on TGV's. One of the points of the present paper, however, is that such an analysis, based on $S, T$ and $U$ is not sufficiently general for inferring the complete low-energy effects of anomalous TGV's. As we have emphasized elsewhere [21], the Peskin-Takeuchi formalism applies only to the extent that new-physics contributions to self-energies for gauge bosons can be approximated as expansions in $q^{2} / M^{2}$, truncated at the linear term. The calculations of ref. [19], as well as our own, indicate that this is not what is obtained through loops from anomalous TGV's. Instead we obtain self-energies of the form

$$
\delta \Pi\left(q^{2}\right)=\omega M_{W}^{2}+\alpha q^{2}+\frac{\beta q^{4}}{M_{W}^{2}}+\frac{\gamma q^{6}}{M_{W}^{4}}
$$

with $\omega, \alpha, \beta$ and $\gamma$ all of the same order of magnitude. The Peskin-Takeuchi parametrization is therefore insufficient, and must be replaced by the more general formalism, recently derived in ref. [21], which involves three additional parameters, denoted $V, W$ and $X$. Our analysis here also differs from that of ref. [19] in that we compute not only the oblique corrections resulting from loop integration, but also corrections to fermion-gauge-boson vertices. These vertex corrections can contribute significantly to low-energy observables.

Our procedure consists of computing how the anomalous TGV's appear in the six observable parameters $S$-X that, in practice, completely parametrize all precision low-

1 For example, $\lambda_{Z}$ and $\Delta \kappa_{Z}$ are the same size in a linearly-realized effective theory. 
energy electroweak measurements. We can then perform a fit, including all charged- and neutral-current data at low energy and at the $Z$ peak. The strength of the conclusions we can draw from such a fit depends crucially on our assumptions regarding which terms we include in our effective lagrangian. If we follow the fairly common practice of fitting for one anomalous TGV at a time, setting the others to zero, we find that that some of the anomalous couplings can be constrained to be too small to detect at LEP200. However, this is rather unrealistic - real models of underlying physics do not generate just one anomalous TGV at a time. A simultaneous fit for the general case in which expressions involving all five anomalous TGV's are fitted to data finds that the constraints are weak, in fact no stronger than those from direct measurements by UA2 [12].

It should be noted that even this is not the least restrictive assumption one can make. Besides the contributions to the parameters $S$ through $X$ that are generated by low-energy loops involving the anomalous TGV's defined at the scale of new physics, $\mu=M$, there are also typically direct contributions to $S$ - $X$ that are generated from $\mathcal{L}_{\text {rest }}$. Obviously, if cancellations are allowed among these two types of new-physics contributions, any constraints on anomalous TGV's are lost. In this sense, current precision electroweak data can never rule out the possibility of anomalous TGV's large enough to be detected at LEP200. Nevertheless, it is a useful exercise to fit for one anomalous TGV at a time, since it does indicate the degree to which the discovery of anomalous TGV's at LEP200 would require cancellations among the new-physics effects for some low-energy observables.

The paper is organized in the following way: In Section 2, we define what we mean by an anomalous TGV lagrangian sector. We imagine that the entire effective lagrangian, defined just below the threshold for new physics, $\mu=M$, consists of the standard model lagrangian plus a supplementary TGV sector. In Section 3, we begin to estimate the indirect effects of this anomalous TGV sector on electroweak observables, by integrating out the physics between $\mu=M$, and the electroweak scale, $\mu \simeq 100 \mathrm{GeV}$. We do so by using the renormalization group $(\mathrm{RG})$ - in the $\overline{\mathrm{MS}}$ renormalization scheme - to run our effective lagrangian between these two scales. We follow in particular how the effective TGV interactions at $\mu=M$ induce other effective interactions at the weak scale, that are detectable in precision electroweak measurements. We call the resulting weak-scale theory the 'grown' lagrangian, and present expressions for the coefficients of the 'grown' operators in terms of the anomalous TGV's. Superficially, this 'grown' lagrangian appears to be complicated, consisting of some 25 differents types of terms.

In Section 4, however, we show that this awkwardness is illusory, since the freedom to redefine fields can be used to greatly reduce the number of terms and to conceptually simplify the calculation of observables. Concretely, this amounts to the use of the SM 
equations of motion to re-express the effective interactions [22]. Exploiting the leeway offered by this technique, we can cast the 'grown' lagrangian into either of two particularly simple forms. On the one hand, we may cast all of the induced interactions as oblique vacuum-polarization corrections, with the proviso that these necessarily involve higherderivative interactions. These make a direct application of the Peskin-Takeuchi $S T U$ formalism invalid, requiring instead the more general analysis in terms of the parameters $S$ through $X$ of ref. [21]. An alternative reformulation, which we use in the present paper, casts the 'grown' lagrangian into a form to which the $S T U$ formalism can be applied directly and which also includes a small number of (higher-derivative) fermion-fermion-gauge-boson interactions that do not contribute to low-energy observables. Both forms are equivalent, and the freedom to transform freely from one to another demonstrates how many terms in the original grown lagrangian ultimately cancel in physical observables. For completeness of presentation, we also give here a very brief summary of the $S T U$ parametrization of new physics, upon which we then build to extend the existing formalism as required by the present analysis. We derive formulae for electroweak observables in terms of the familiar $S, T, U$ and the new parameters $V, W, X$.

In Section 5 we then present expressions for these six parameters in terms of the anomalous TGV couplings. We explain how these expressions can be used to place phenomenological bounds on the TGV couplings and we conclude that such bounds are weak. In Section 6 we present the results of fits to these expressions. We first consider the limit in which each TGV is separately turned on at $\mu=M$, with all other effective interactions being zero. We find that, with this somewhat unphysical assumption, the data place strong phenomenological bounds on the TGV couplings that are of order of several percent. However, when we fit for the five anomalous TGV's simultaneously, we find that the bounds become considerably weaker, of $\mathcal{O}(1)$.

Finally, in Section 7 we discuss the results, commenting in particular on some rather startling cancellations in our final expressions. These cancellations decrease the sensitivity of some observables to certain anomalous TGV's.

\section{The Anomalous TGV's}

Let us start by considering the effective lagrangian, as defined at the scale of new physics, $\mu=M$, after the lightest of the heavy new particles has been integrated out. In general many effective interactions appear in this lagrangian, but we wish to focus in this 
paper only on a few of these ${ }^{2}$ :

$$
\mathcal{L}=\mathcal{L}_{S M}\left(\tilde{e}_{i}\right)+\mathcal{L}_{T G V}
$$

where $\mathcal{L}_{S M}\left(\tilde{e}_{i}\right)$ is the standard model (SM) lagrangian, in which the as-yet-undetected particles - in particular, the Higgs boson - have been integrated out. We place tildes on the three electroweak parameters $\left(\tilde{e}_{i} \equiv\left\{\tilde{e}, \tilde{s}_{w}\left(\equiv \sin \tilde{\theta}_{W}\right), \tilde{m}_{Z}\right\}\right)$ that appear in this lagrangian as a reminder that their values have been shifted from their 'standard' values, which we denote without tildes: $e, s_{w}, m_{z}$. These 'standard' values are the ones that are obtained by fitting radiatively corrected SM expressions for observables to precise data.

$\mathcal{L}_{T G V}$ contains the anomalous CP-conserving TGV's which are the focus of this study. Following refs. [1] and [23] we write:

$$
\begin{aligned}
\mathcal{L}_{T G V}=i g_{Z} \Delta & g_{1 z}\left(W_{\alpha \beta} W^{* \beta}-W_{\alpha \beta}^{*} W^{\beta}\right) Z^{\alpha} \\
& +i \sum_{V=Z, \gamma}\left[g_{V} \Delta \kappa_{V} W_{\alpha}^{*} W_{\beta} V^{\alpha \beta}+g_{V} \frac{\lambda_{V}}{M_{W}^{2}} W_{\rho \mu}^{*} W^{\mu}{ }_{\sigma} V^{\sigma \rho}\right] .
\end{aligned}
$$

Here $g_{\gamma}=e$ and $g_{z}=e c_{w} / s_{w}$ denote the SM couplings. Electromagnetic gauge invariance requires that $\Delta g_{1 \gamma}=0$. A gauge field having two lorentz indices, such as $W_{\alpha \beta}$, denotes the abelian curl of the corresponding gauge potential, defined using the appropriate electromagnetic gauge-covariant derivative: $W_{\alpha \beta}=D_{\alpha} W_{\beta}-D_{\beta} W_{\alpha}$. Here $D_{\alpha} W_{\beta}=\partial_{\alpha} W_{\beta}+i e A_{\alpha} W_{\beta}$. Following the convention established in the literature, we scale the $\lambda_{V}$ term to $M_{W}^{2}$, despite the fact that it is a dimension-six operator and should more correctly have a factor $M^{2}$ in the denominator. Due to the appearance of the $U_{\text {em }}(1)$-covariant derivatives, $\mathcal{L}_{T G V}$ also contains four- and five-point gauge-boson vertices:

$$
\mathcal{L}_{T G V}=\mathcal{L}_{3 B}+\mathcal{L}_{4 B}+\mathcal{L}_{5 B}
$$

where $\mathcal{L}_{3 B}$ is simply $\mathcal{L}_{T G V}$ with the replacement $D_{\mu} \rightarrow \partial_{\mu}$, and $\mathcal{L}_{4 B}$ is given by

$$
\mathcal{L}_{4 B}=e g_{z} \Delta g_{1 z}\left[W_{\alpha}^{*} W_{\beta}\left(Z^{\alpha} A^{\beta}+Z^{\beta} A^{\alpha}\right)-2 W_{\alpha}^{*} W^{\alpha} A_{\beta} Z^{\beta}\right]+\left(\lambda_{V} \text { terms }\right)
$$

The ' $\lambda_{V}$ terms' here consist of those four-point interactions that are generated from the $\lambda_{V}$ terms in $\mathcal{L}_{T G V}$. We do not list them explicitly because, for present purposes, all graphs

2 Note that the terms we ignore include direct new-physics contributions to $S, T$ and $U$ [9], as well as to gauge-boson-fermion-fermion and other vertices. 
which use these rules turn out to vanish. As for $\mathcal{L}_{5 B}$, such terms contribute only in two-loop diagrams and are ignored here.

\section{Loop Integration and the Low-Energy Lagrangian}

We next calculate the loop effects of the anomalous TGV's of the previous section. We do so by running the effective interactions of the lagrangian defined at the new-physics

scale, $\mu \simeq M$, down to the weak scale, $\mu \simeq M_{Z}$, where we can extract their consequences for precision experiments. We compute here the coefficients of the effective interactions at the weak scale that are generated through this renormalization group (RG) mixing with the operators in $\mathcal{L}_{T G V}$.

\section{1) Vacuum Polarization and Vertex Corrections}

The required diagrams fall into two categories: contributions to the gauge-boson vacuum polarization — i.e. 'oblique' - corrections, as shown in Fig. (1), and fermion-gaugeboson vertex corrections, as shown in Fig. (2). We define the running of our operators using the $\overline{\mathrm{MS}}$ renormalization scheme. Here we simply quote the results, presenting only the coefficient of $\left[2 /(4-n)-\gamma_{E}+\ln \left(4 \pi \mu^{2}\right)\right]$, where $n$ is the dimension of spacetime.

Evaluating the graphs of Fig. (1), we find the following TGV-induced contributions to the gauge-boson vacuum polarization tensors. With the definition

$$
\delta \Pi_{a b}^{\mu \nu}(q)=\eta^{\mu \nu} \delta \Pi_{a b}\left(q^{2}\right)+q^{\mu} q^{\nu} \quad \text { terms }
$$

where $a, b=W, Z$ and $\gamma$, the coefficient of $\left[2 /(4-n)-\gamma_{E}+\ln \left(4 \pi \mu^{2}\right)\right]$ in $\delta \Pi_{a b}\left(q^{2}\right)$ is:

$$
\begin{aligned}
\delta \Pi_{\gamma \gamma}\left(q^{2}\right) & =\alpha_{\gamma} q^{2}+\beta_{\gamma} \frac{q^{4}}{M_{z}^{2}}+\gamma_{\gamma} \frac{q^{6}}{M_{z}^{4}} \\
\delta \Pi_{Z \gamma}\left(q^{2}\right) & =\alpha_{z \gamma} q^{2}+\beta_{z \gamma} \frac{q^{4}}{M_{z}^{2}}+\gamma_{z \gamma} \frac{q^{6}}{M_{z}^{4}} \\
\delta \Pi_{W W}\left(q^{2}\right) & =\omega_{W} M_{W}^{2}+\alpha_{W} q^{2}+\beta_{W} \frac{q^{4}}{M_{W}^{2}}+\gamma_{W} \frac{q^{6}}{M_{W}^{4}} \\
\delta \Pi_{Z Z}\left(q^{2}\right) & =\omega_{z} M_{z}^{2}+\alpha_{z} q^{2}+\beta_{z} \frac{q^{4}}{M_{z}^{2}}+\gamma_{z} \frac{q^{6}}{M_{z}^{4}},
\end{aligned}
$$

where $\alpha_{z}, \beta_{\gamma}$ etc. are given as functions of the couplings $\Delta g_{1 z}, \Delta \kappa_{V}$ and $\lambda_{V}$ in Table I. Anticipating that these anomalous couplings are small, we drop all terms past linear 


\begin{tabular}{|c|c|}
\hline Coefficient & One-Loop Result $\times\left(\alpha / 4 \pi s_{w}^{2}\right) \log \left(\mu^{\prime 2} / \mu^{2}\right)$ \\
\hline $\begin{array}{l}\alpha_{\gamma}\left(\mu^{2}\right) \\
\beta_{\gamma}\left(\mu^{2}\right) \\
\gamma_{\gamma}\left(\mu^{2}\right) \\
\alpha_{z \gamma}\left(\mu^{2}\right) \\
\beta_{z \gamma}\left(\mu^{2}\right) \\
\gamma_{z \gamma}\left(\mu^{2}\right) \\
\omega_{W}\left(\mu^{2}\right) \\
\alpha_{W}\left(\mu^{2}\right) \\
\beta_{W}\left(\mu^{2}\right) \\
\gamma_{W}\left(\mu^{2}\right) \\
\omega_{Z}\left(\mu^{2}\right) \\
\alpha_{z}\left(\mu^{2}\right) \\
\beta_{z}\left(\mu^{2}\right) \\
\gamma_{z}\left(\mu^{2}\right)\end{array}$ & $\begin{array}{c}s_{w}^{2}\left(6 \Delta \kappa_{\gamma}-12 \lambda_{\gamma}\right) \\
s_{w}^{2}\left(-\frac{2}{3} \Delta \kappa_{\gamma}+2 \lambda_{\gamma}\right) / c_{w}^{2} \\
-\frac{1}{6} s_{w}^{2} \Delta \kappa_{\gamma} / c_{w}^{4} \\
c_{w} s_{w}\left[3 \Delta \kappa_{Z}+3 \Delta \kappa_{\gamma}-6 \lambda_{z}-6 \lambda_{\gamma}+4 \Delta g_{1 z}\right] \\
\left(s_{w} / c_{w}\right)\left[-\frac{1}{3}\left(\Delta \kappa_{Z}+\Delta \kappa_{\gamma}\right)+\lambda_{Z}+\lambda_{\gamma}-\frac{5}{6} \Delta g_{1 z}\right] \\
-\left(s_{w} / 12 c_{w}^{3}\right)\left[\Delta \kappa_{Z}+\Delta \kappa_{\gamma}\right] \\
\left.\frac{5}{3} s_{w}^{2} \Delta \kappa_{\gamma}+\frac{1}{3}\left(7+5 c_{w}^{2}\right) \Delta \kappa_{z}-6 s_{w}^{2} \lambda_{\gamma}-6\left(1+c_{w}^{2}\right) \lambda_{z}+\frac{1}{2}\left(1+\frac{1}{2} c_{w}^{4}\right) \Delta g_{1 z}^{2}+\frac{17}{3} c_{w}^{4}\right) \Delta g_{1 z} \\
{\left[-\frac{5}{6}\left(s_{w}^{2} \Delta \kappa_{\gamma}+c_{w}^{2} \Delta \kappa_{Z}\right)+2\left(s_{w}^{2} \lambda_{\gamma}+c_{w}^{2} \lambda_{Z}\right)-\frac{1}{6}\left(2 c_{w}^{2}+7 c_{w}^{4}\right) \Delta g_{1 z}\right]} \\
-\frac{1}{6} c_{w}^{4} \Delta g_{1 z} \\
9 c_{w}^{4} \Delta g_{1 z} \\
c_{w}^{2}\left[6 \Delta \kappa_{Z}-12 \lambda_{z}+8 \Delta g_{1 z}\right] \\
{\left[-\frac{2}{3} \Delta \kappa_{Z}+2 \lambda_{Z}-\frac{5}{3} \Delta g_{1 z}\right]} \\
-\left(1 / 6 c_{w}^{2}\right) \Delta \kappa_{Z}\end{array}$ \\
\hline
\end{tabular}

TABLE I

One-loop results for the coefficients in the gauge-boson vacuum polarization in terms of the various TGV couplings, where the TGV couplings are defined at scale $\mu^{\prime}$.

order in the expressions in the table. The vacuum polarization diagrams have also been calculated by the authors of ref. [19], and our results are in agreement with theirs for diagrams involving $\Delta \kappa_{V}$ and $\lambda_{V}$. They did not calculate the diagrams with $\Delta g_{1 z}$.

As for the vertex graphs of Fig. (2), we obtain the following expressions for the $[2 /(4-$ $\left.n)-\gamma_{E}+\ln \left(4 \pi \mu^{2}\right)\right]$ coefficient in the fermion-fermion-gauge-boson vertex corrections:

$$
\begin{aligned}
\delta \Lambda_{\mathrm{em}}\left(q^{2}\right) & =\left(p_{\mathrm{em}}^{(2)} \frac{q^{2}}{M_{Z}^{2}}+p_{\mathrm{em}}^{(4)} \frac{q^{4}}{M_{Z}^{4}}\right) T_{3 f} \gamma_{L} \\
\delta \Lambda_{\mathrm{cc}}\left(q^{2}\right) & =\left(p_{\mathrm{cc}}^{(0)}+p_{\mathrm{cc}}^{(2)} \frac{q^{2}}{M_{W}^{2}}+p_{\mathrm{cc}}^{(4)} \frac{q^{4}}{M_{W}^{4}}\right) V_{f f^{\prime}} \gamma_{L} \\
\delta \Lambda_{\mathrm{nc}}\left(q^{2}\right) & =\left(p_{\mathrm{nc}}^{(2)} \frac{q^{2}}{M_{Z}^{2}}+p_{\mathrm{nc}}^{(4)} \frac{q^{4}}{M_{Z}^{4}}\right) T_{3 f} \gamma_{L} .
\end{aligned}
$$

where we have normalized the vertex corrections such that standard model tree-level ver- 
tices are corrected in the following manner:

$$
\begin{aligned}
i \Lambda_{\mathrm{em}}^{\mu}\left(q^{2}\right) & =-i e \gamma^{\mu}\left[Q_{f}+\frac{1}{s_{w}} \delta \Lambda_{\mathrm{em}}\left(q^{2}\right)\right] \\
i \Lambda_{\mathrm{cc}}^{\mu}\left(q^{2}\right) & =-i \frac{e}{\sqrt{2} s_{w}} \gamma^{\mu}\left[V_{f f^{\prime}} \gamma_{L}+\delta \Lambda_{\mathrm{cc}}\left(q^{2}\right)\right] \\
i \Lambda_{\mathrm{nc}}^{\mu}\left(q^{2}\right) & =-i \frac{e}{s_{w} c_{w}} \gamma^{\mu}\left[T_{3 f} \gamma_{L}-Q_{f} s_{w}^{2}+c_{w} \delta \Lambda_{\mathrm{nc}}\left(q^{2}\right)\right]
\end{aligned}
$$

where $f, f^{\prime}$ denote fermion type, $T_{3 f}$ is the weak isospin, and $Q_{f}$ the electric charge. In the charged-current expression, $V_{f f^{\prime}}$ represents the usual Cabibbo-Kobayashi-Maskawa $(\mathrm{CKM})$ matrix in generation space when the external fermions are quarks, and is given by $\delta_{f f^{\prime}}$ when they are leptons.

We give expressions for the $p^{(n)}$ coefficients as linear combinations of the anomalous TGV couplings in Table II. Notice that since we have calculated in the approximation that all fermions are massless, we have 'grown' only left-handed corrections to the standard model fermion-fermion-gauge-boson couplings.

\begin{tabular}{lc}
\hline \hline Coefficient & One-Loop Result $\times\left(\alpha / 4 \pi s_{w}^{2}\right) \log \left(\mu^{\prime 2} / \mu^{2}\right)$ \\
\hline$p_{\text {nc }}^{(2)}\left(\mu^{2}\right)$ & $\left(1 / 2 c_{w}\right)\left[\Delta \kappa_{Z}-2 \lambda_{z}+\frac{5}{3} \Delta g_{1 z}\right]$ \\
$p_{\text {nc }}^{(4)}\left(\mu^{2}\right)$ & $\left(1 / 12 c_{w}^{3}\right) \Delta \kappa_{Z}$ \\
$p_{\text {cc }}^{(0)}\left(\mu^{2}\right)$ & $\frac{3}{4}\left[\left(c_{w}^{2}-1\right) \Delta \kappa_{Z}+s_{w}^{2} \Delta \kappa_{\gamma}+\left(c_{w}^{2}-c_{w}^{4}\right) \Delta g_{1 z}\right]$ \\
$p_{\text {cc }}^{(2)}\left(\mu^{2}\right)$ & $\frac{1}{4}\left[\frac{5}{3}\left(c_{w}^{2} \Delta \kappa_{Z}+s_{w}^{2} \Delta \kappa_{\gamma}\right)-4\left(c_{w}^{2} \lambda_{z}+s_{w}^{2} \lambda_{\gamma}\right)+\left(c_{w}^{2}+\frac{8}{3} c_{w}^{4}\right) \Delta g_{1 z}\right]$ \\
$p_{\text {cc }}^{(4)}\left(\mu^{2}\right)$ & $\frac{1}{12} c_{w}^{4} \Delta g_{1 z}$ \\
$p_{\text {em }}^{(2)}\left(\mu^{2}\right)$ & $\left(s_{w} / 2 c_{w}^{2}\right)\left[\Delta \kappa_{\gamma}-2 \lambda_{\gamma}\right]$ \\
$p_{\mathrm{em}}^{(4)}\left(\mu^{2}\right)$ & $\left(s_{w} / 12 c_{w}^{4}\right) \Delta \kappa_{\gamma}$ \\
\hline \hline
\end{tabular}

\section{TABLE II}

One-loop results for the coefficients in the gauge-boson-fermion vertex corrections in terms of the various TGV couplings.

The above expressions are universal corrections because of our neglect of all fermion masses. However, the one situation for which this assumption is inadequate is the coupling of the down-type quarks to the photon and to the $Z$ boson, since these involve virtual top 
quarks, whose mass is not small. ${ }^{3}$ Only the $Z b \bar{b}$ vertex is of practical importance, though, because the only process in which these interactions are probed is in the decay of the $Z$ into $b \bar{b}$ pairs. For this observable the vertex correction has the form:

$$
\delta \Lambda_{\mathrm{nc}}^{b \bar{b}}\left(q^{2}\right)=\delta \Lambda_{\mathrm{nc}}^{\text {univ }}\left(q^{2}\right)+\frac{1}{64 \pi^{2}}\left|V_{t b}\right|^{2} \frac{e^{2} c_{w}}{s_{w}^{2}}\left(\frac{m_{t}^{2}}{M_{W}^{2}}\right) \log \left(\frac{\mu^{\prime 2}}{\mu^{2}}\right)\left[3 \Delta g_{1 z}+\frac{1}{2 c_{w}^{2}} \Delta \kappa_{Z}\left(\frac{q^{2}}{M_{Z}^{2}}\right)\right] \gamma_{L}
$$

where $\delta \Lambda_{\mathrm{nc}}^{\text {univ. }}\left(q^{2}\right)$ is the result given in eq. (9) and Table II.

\section{2) The Weak-Scale Effective Lagrangian}

These expressions may be interpreted as contributions to the effective lagrangian at lower energy scales. In the $\overline{\mathrm{MS}}$ scheme the resulting expressions for the induced couplings at scale $\mu$ may be obtained from the table by simply multiplying the results of Tables I and II by $y \equiv \ln \left(\left(\mu^{\prime} / \mu\right)^{2}\right)$, where the TGV couplings $\Delta \kappa_{z}, \Delta \kappa_{\gamma}$ etc. are taken to be defined at scale $\mu^{\prime}$.

We may therefore write out those terms that are 'grown' in the low-energy lagrangian at scale $\mu$, due to the appearance of $\mathcal{L}_{T G V}$ at the higher scale $\mu^{\prime}=M$ :

$$
\frac{1}{y} \mathcal{L}_{\text {grown }}=\mathcal{L}_{\text {vac. pol. }}+\mathcal{L}_{\text {ver. corr. }}+\mathcal{L}_{\text {nonuniv. }}
$$

with $\mathcal{L}_{\text {nonuniv. }}$ containing the non-universal $m_{t}$-dependent contributions of eq. (11), while

$$
\begin{aligned}
\mathcal{L}_{\text {vac. pol. }}=\frac{\omega_{Z} M_{Z}^{2}}{2} Z_{\mu} Z^{\mu}+\frac{\alpha_{Z}}{4} Z_{\mu \nu} Z^{\mu \nu}-\frac{\beta_{Z}}{4 M_{Z}^{2}} Z_{\mu \nu} \square Z^{\mu \nu}+\frac{\gamma_{Z}}{4 M_{Z}^{4}} Z_{\mu \nu} \square^{2} Z^{\mu \nu} \\
\quad+\frac{\alpha_{Z \gamma}}{2} Z_{\mu \nu} F^{\mu \nu}-\frac{\beta_{Z \gamma}}{2 M_{Z}^{2}} Z_{\mu \nu} \square F^{\mu \nu}+\frac{\gamma_{z \gamma}}{2 M_{Z}^{4}} Z_{\mu \nu} \square^{2} F^{\mu \nu} \\
+\frac{\alpha_{\gamma}}{4} F_{\mu \nu} F^{\mu \nu}-\frac{\beta_{\gamma}}{4 M_{Z}^{2}} F_{\mu \nu} \square F^{\mu \nu}+\frac{\gamma_{\gamma}}{4 M_{Z}^{4}} F_{\mu \nu} \square^{2} F^{\mu \nu} \\
+\omega_{W} M_{W}^{2} W_{\mu}^{*} W^{\mu}+\frac{\alpha_{W}}{2} W_{\mu \nu}^{*} W^{\mu \nu}-\frac{\beta_{W}}{2 M_{W}^{2}} W_{\mu \nu}^{*} \square W^{\mu \nu}+\frac{\gamma_{W}}{2 M_{W}^{4}} W_{\mu \nu}^{*} \square^{2} W^{\mu \nu}
\end{aligned}
$$

3 This necessity to keep track of the top-quark mass is one of the differences between our calculation and that of ref. [17], who find that the fermion masses do not affect the $\overline{\mathrm{MS}} \mathrm{RG}$ evolution at the one-loop level in the linearly-realized effective theory. 
and

$$
\begin{aligned}
\mathcal{L}_{\text {ver. corr. }}=( & \left.s_{w} j^{\mu}+c_{w} N^{\mu}\right)\left(-p_{\mathrm{nc}}^{(2)} \frac{\square}{M_{Z}^{2}}+p_{\mathrm{nc}}^{(4)} \frac{\square^{2}}{M_{Z}^{4}}\right) Z_{\mu} \\
+ & \left(s_{w} j^{\mu}+c_{w} N^{\mu}\right)\left(-p_{\mathrm{em}}^{(2)} \frac{\square}{M_{Z}^{2}}+p_{\mathrm{em}}^{(4)} \frac{\square^{2}}{M_{Z}^{4}}\right) A_{\mu} \\
+ & {\left[J^{\mu}\left(p_{\mathrm{cc}}^{(0)}-p_{\mathrm{cc}}^{(2)} \frac{\square}{M_{W}^{2}}+p_{\mathrm{cc}}^{(4)} \frac{\square^{2}}{M_{W}^{4}}\right) W_{\mu}^{*}+\text { h.c. }\right] . }
\end{aligned}
$$

Here $j^{\mu}, N^{\mu}$ and $J^{\mu}$ are respectively the total SM electromagnetic, neutral and charged currents:

$$
\begin{aligned}
j^{\mu} & =-e \sum_{f} \bar{\Psi}_{f} \gamma^{\mu} Q_{f} \Psi_{f}, \\
J^{\mu} & =-\frac{e}{\sqrt{2} s_{w}} \sum_{f f^{\prime}} \bar{\Psi}_{f} \gamma^{\mu} V_{f f^{\prime}} \gamma_{L} \Psi_{f^{\prime}}, \\
N^{\mu} & =-\frac{e}{s_{w} c_{w}} \sum_{f} \bar{\Psi}_{f} \gamma^{\mu}\left[T_{3 f} \gamma_{L}-Q_{f} s_{w}^{2}\right] \Psi_{f},
\end{aligned}
$$

so that $\mathcal{L}_{S M}=j^{\mu} A_{\mu}+N^{\mu} Z_{\mu}+\left(J^{\mu} W_{\mu}^{*}+\right.$ h.c. $)+\ldots$

To the extent that the loop effects of anomalous TGV's are universal in their coupling to fermion generations, we see that it is possible to express the 'grown' fermion-gauge couplings in terms of linear combinations of total SM currents. This is important, since it in turn allows - through the use of equations of motion - a significant simplification of the effective lagrangian. The exception to this universal form is in the $m_{t}$-dependent interactions of $\mathcal{L}_{\text {nonuniv., }}$ in which the $Z$ boson and the photon do not couple with the same strength to all generations of down quarks. This particular case must be treated separately, but does not affect the arguments of the subsequent sections.

\section{Simplifying the Effective Theory}

At this point one might be daunted by the fact that there are no fewer than 29 types of terms in this 'grown' lagrangian, since this potentially makes any further analysis very cumbersome. This complicated form for $\mathcal{L}_{\text {grown }}$ is illusory, however, since not all of the effective interactions displayed in Eqs. (13) and (14) are independent of one another. The point here is that, due to the freedom to perform field redefinitions, there are a great many ways of writing any particular term in an effective lagrangian. In particular, any two 
operators which can be transformed into one another using the SM equations of motion may also be transformed into one another by a field redefinition [22].

We may therefore use the SM equations of motion to simplify $\mathcal{L}_{\text {grown }}$, with two goals in mind. We wish to firstly reduce the number of types of terms that we must consider. Next, in choosing which of the remaining terms to keep as a basis of independent interactions, we wish to take advantage, as much as possible, of existing formalism and bounds on various types of new physics that have been obtained by previous workers. We therefore try, in particular, to cast the grown lagrangian into a form that is amenable to an application of the $S T U$ formalism of Peskin and Takeuchi [9]. As we shall see, this is not completely possible, due to the appearance in $\mathcal{L}_{\text {grown }}$ of higher-derivative interactions, and so a naive application of this formalism turns out to be incorrect. Instead, along the lines of [21], we use an extension of this formalism that permits the treatment of more general $q^{2}$ dependence.

\section{1) STU Formalism}

Before casting the 'grown' lagrangian in a form amenable to an application of the $S T U$ formalism, let us rapidly review the $S T U$ basics, following a method that we have developed in ref. [24].

Imagine supplementing the standard model by an 'STU-sector', i.e. by the lowestdimension effective two-point interactions for gauge bosons:

$$
\begin{aligned}
\mathcal{L}_{e f f}= & \mathcal{L}_{S M}\left(\tilde{e}_{i}\right)+\mathcal{L}_{S T U}, \\
\text { with } \quad \mathcal{L}_{S T U}=- & \frac{A}{4} F_{\mu \nu} F^{\mu \nu}-\frac{B}{2} W_{\mu \nu}^{*} W^{\mu \nu}-\frac{C}{4} Z_{\mu \nu} Z^{\mu \nu}+\frac{G}{2} F_{\mu \nu} Z^{\mu \nu} \\
& +w \tilde{m}_{W}^{2} W_{\mu}^{*} W^{\mu}+\frac{z}{2} \tilde{m}_{Z}^{2} Z_{\mu} Z^{\mu} .
\end{aligned}
$$

where $\tilde{e}_{i}$ are defined as in Eq. (3). This parametrization implies that self-energies generated by new physics are of the form

$$
\delta \Pi_{a}\left(q^{2}\right)=\omega_{a}+\alpha_{a} q^{2}
$$

which is consistent with the idea that $q^{2} \ll M^{2}$ (where $M$ is the scale of new physics) and that $\delta \Pi_{a}\left(q^{2}\right)$ can be expanded as a Taylor series in $q^{2} / M^{2}$, truncated at the linear term.

It is a simple matter to canonically normalize and diagonalize the gauge-boson kinetic terms. Working to linear order in the small coefficients $A, B, \ldots$, the required field 
redefinitions are

$$
\begin{aligned}
A_{\mu} & \rightarrow\left(1-\frac{A}{2}\right) A_{\mu}+G Z_{\mu} \\
W_{\mu} & \rightarrow\left(1-\frac{B}{2}\right) W_{\mu} \\
Z_{\mu} & \rightarrow\left(1-\frac{C}{2}\right) Z_{\mu} .
\end{aligned}
$$

The field transformations affect the electromagnetic, charged and neutral current couplings and the mass terms, which now become:

$$
\begin{aligned}
\mathcal{L}_{\mathrm{em}} & =-\tilde{e}\left(1-\frac{A}{2}\right) \sum_{f} \bar{\Psi}_{f} \gamma^{\mu} Q_{f} \Psi_{f} A_{\mu}, \\
\mathcal{L}_{\mathrm{cc}} & =-\frac{\tilde{e}}{\tilde{s}_{w} \sqrt{2}}\left(1-\frac{B}{2}\right) \sum_{f f^{\prime}} \widetilde{V}_{f f^{\prime}} \bar{\Psi}_{f} \gamma^{\mu} \gamma_{L} \Psi_{f^{\prime}} W_{\mu}^{*}+h . c . \\
\mathcal{L}_{\mathrm{nc}} & =-\frac{\tilde{e}}{\tilde{s}_{w} \tilde{c}_{w}}\left(1-\frac{C}{2}\right) \sum_{f} \bar{\Psi}_{f} \gamma^{\mu}\left[T_{3 f} \gamma_{L}-Q_{f} \tilde{s}_{w}^{2}+Q_{f} \tilde{s}_{w} \tilde{c}_{w} G\right] \Psi_{f^{\prime}} Z_{\mu}, \\
\mathcal{L}_{W, Z} & =(1+w-B) \tilde{m}_{W}^{2} W_{\mu}^{*} W^{\mu}+\frac{1}{2}(1+z-C) \tilde{m}_{z}^{2} Z_{\mu} Z^{\mu} .
\end{aligned}
$$

Our next step is to eliminate $\tilde{e}_{i}$ in favour of the 'standard' parameters $e_{i}$. To this end, we express our model's predictions for the three precisely measured observables $\alpha, G_{F}$ and $M_{Z}$ and equate these expressions to the standard model formulae:

$$
\begin{aligned}
4 \pi \alpha & =4 \pi \alpha_{S M}\left(\tilde{e}, \tilde{s}_{w}, \tilde{m}_{Z}\right)(1-A) \equiv 4 \pi \alpha_{S M}\left(e, s_{w}, m_{Z}\right), \\
G_{F} & =G_{F S M}\left(\tilde{e}, \tilde{s}_{w}, \tilde{m}_{Z}\right)(1-w) \equiv G_{F S M}\left(e, s_{w}, m_{Z}\right), \\
M_{Z}^{2} & =M_{Z S M}^{2}\left(\tilde{e}, \tilde{s}_{w}, \tilde{m}_{Z}\right)(1+z-C) \equiv M_{Z S M}^{2}\left(e, s_{w}, m_{Z}\right) .
\end{aligned}
$$

Inverting these equations, we obtain

$$
\begin{aligned}
\tilde{e} & =e\left(1+\frac{A}{2}\right), \\
\tilde{m}_{z}^{2} & =m_{z}^{2}(1-z+C), \\
\tilde{s}_{w}^{2} & =s_{w}^{2}\left(1-\frac{c_{w}^{2}}{c_{w}^{2}-s_{w}^{2}}(-A-z+C+w)\right) .
\end{aligned}
$$


With the substitution of Eqs. (28) into the various lagrangian terms given in Eqs. (23), (24) and (25), one obtains interaction terms consisting of the usual standard model piece corrected by a linear combination of the parameters $A$ through $z$. It turns out, though, that these parameters can only appear in three linearly independent combinations, a convenient choice being

$$
\begin{aligned}
\alpha S & =4 s_{w}^{2} c_{w}^{2}(A-C)-4 s_{w} c_{w}\left(c_{w}^{2}-s_{w}^{2}\right) G, \\
\alpha T & =w-z, \\
\alpha U & =4 s_{w}^{2}\left(s_{w}^{2} A-B+c_{w}^{2} C-2 s_{w} c_{w} G\right),
\end{aligned}
$$

so that all the corrections can be expressed in terms of $S, T$ and $U$. After this algorithm has been performed, the interaction and $W$ boson mass terms become:

$$
\begin{aligned}
& \mathcal{L}_{\mathrm{cc}}=-\frac{e}{\sqrt{2} s_{w}}\left(1-\frac{\alpha S}{4\left(c_{w}^{2}-s_{w}^{2}\right)}+\frac{c_{w}^{2} \alpha T}{2\left(c_{w}^{2}-s_{w}^{2}\right)}+\frac{\alpha U}{8 s_{w}^{2}}\right) \sum_{f f^{\prime}} V_{f f^{\prime}} \bar{\Psi}_{f} \gamma^{\mu} \gamma_{L} \Psi_{f^{\prime}} W_{\mu}^{*}+h . c .(30) \\
& \mathcal{L}_{\mathrm{nc}}=-\frac{e}{s_{w} c_{w}}\left(1+\frac{\alpha T}{2}\right) \sum_{f} \bar{\Psi}_{f} \gamma^{\mu}\left[T_{3 f} \gamma_{L}-Q_{f}\left(s_{w}^{2}+\frac{\alpha S}{4\left(c_{w}^{2}-s_{w}^{2}\right)}-\frac{c_{w}^{2} s_{w}^{2} \alpha T}{c_{w}^{2}-s_{w}^{2}}\right)\right] \Psi_{f} Z_{\mu}(31) \\
& \mathcal{L}_{W}=m_{z}^{2} c_{w}^{2}\left[1-\frac{\alpha S}{2\left(c_{w}^{2}-s_{w}^{2}\right)}+\frac{c_{w}^{2} \alpha T}{c_{w}^{2}-s_{w}^{2}}+\frac{\alpha U}{4 s_{w}^{2}}\right] W_{\mu}^{*} W^{\mu} .
\end{aligned}
$$

From Eqs. (30), (31) and (32), it is a simple matter to compute the $S T U$ dependence in expressions for various electroweak observables. Such expressions can then be compared to experimental data in order to place constraints on $S, T$ and $U$. These constraints can be translated into bounds on the parameters of the new physics in terms of which $S, T$ and $U$ are computed.

However, this formalism cannot be directly applied in the present TGV analysis: as mentioned earlier, it is based on the assumption that $\delta \Pi\left(q^{2}\right)$ can be expressed as a Taylor series in $q^{2} / M^{2}$ truncated at the linear term, whereas in the present anomalous TGV application (with non-linearly realized gauge-symmetry) the new physics self-energies are of the form

$$
\delta \Pi_{W}\left(q^{2}\right)=\omega M_{W}^{2}+\alpha q^{2}+\frac{\beta q^{4}}{M_{W}^{2}}+\frac{\gamma q^{6}}{M_{W}^{4}}
$$

with $\omega, \alpha, \beta$ and $\gamma$ all of the same order. Clearly the $S T U$ formalism as it stands cannot be applied directly, a point which is easy to miss if the definitions given in ref. [10] are used:

$$
\alpha S=\frac{4}{M_{Z}^{2}}\left[c_{w}^{2} s_{w}^{2}\left(\Pi_{Z Z}\left(M_{Z}^{2}\right)-\Pi_{Z Z}(0)\right)\right.
$$




$$
\begin{aligned}
& \left.-c_{w}^{2} s_{w}^{2} \Pi_{\gamma \gamma}\left(M_{Z}^{2}\right)+s_{w} c_{w}\left(s_{w}^{2}-c_{w}^{2}\right) \Pi_{Z \gamma}\left(M_{Z}^{2}\right)\right], \\
\alpha T & =\frac{\Pi_{W W}(0)}{M_{W}^{2}}-\frac{\Pi_{Z Z}(0)}{M_{Z}^{2}}, \\
\alpha U & =4 s_{w}^{2}\left[\frac{1}{M_{W}^{2}}\left(\Pi_{W W}\left(M_{W}^{2}\right)-\Pi_{W W}(0)\right)\right. \\
& \left.-\frac{1}{M_{Z}^{2}}\left(s_{w}^{2} \Pi_{\gamma \gamma}\left(M_{Z}^{2}\right)+c_{w}^{2}\left(\Pi_{Z Z}\left(M_{Z}^{2}\right)-\Pi_{Z Z}(0)\right)+2 c_{w} s_{w} \Pi_{Z \gamma}\left(M_{Z}^{2}\right)\right)\right] .
\end{aligned}
$$

The point is that although these definitions make sense for arbitrary vacuum polarizations, it turns out that it is impossible to express all well-measured observables using just these three parameters, unless the self-energy expansions are truncated at linear order in $q^{2}$. A method for dealing with the case of general $q^{2}$ dependence in the vacuum polarization is given in ref. [21], where it is shown that, in practice, the contributions to current precision electroweak measurements may be encapsulated into only six variables, called $(S T U V W X)$.

We now turn to the task of casting our effective lagrangian in the form most amenable to an application of the methods presented above.

\section{2) Using Equations of Motion to Simplify the Effective Lagrangian}

As was mentioned earlier, the analysis presented in Section 3 seems somewhat cluttered, since $\mathcal{L}_{\text {grown }}$ entails 29 types of terms, including oblique corrections (dimension-six and -eight terms) as well as vertex corrections. Moreover, the presence of higher-derivative operators appears to hinder the direct application of the formalism presented in the previous subsection. So, to overcome these difficulties, we exploit the freedom to simplify these interactions using the Euler-Lagrange equations of motion derived from $\mathcal{L}_{S M}\left(\tilde{e}_{i}\right)$,

$$
\begin{aligned}
\partial_{\mu}\left(\partial^{\mu} A^{\nu}-\partial^{\nu} A^{\mu}\right) & =-\tilde{j}^{\nu}, \\
\partial_{\mu}\left(\partial^{\mu} Z^{\nu}-\partial^{\nu} Z^{\mu}\right) & =-\tilde{N}^{\nu}-\tilde{m}_{Z}^{2} Z^{\nu}, \\
\partial_{\mu}\left(\partial^{\mu} W^{\nu}-\partial^{\nu} W^{\mu}\right) & =-\tilde{J}^{\nu}-\tilde{m}_{W}^{2} W^{\nu} .
\end{aligned}
$$

Moreover, one can also make liberal use of the freedom to integrate by parts to relate otherwise disparate operators.

It is useful to note that a certain leeway exists in transforming operators with the above-mentioned methods. For example, a general re-expression of the operator $Z_{\mu \nu} \square Z^{\mu \nu}$ 
would be

$$
\begin{aligned}
Z_{\mu \nu} \square Z^{\mu \nu} \rightarrow & -\eta \rho M_{Z}^{2} Z_{\mu \nu} Z^{\mu \nu}-2 \eta(1-\rho) M_{Z}^{4} Z_{\mu} Z^{\mu}-2 \eta(2-\rho-\xi) M_{Z}^{2} Z_{\mu} N^{\mu} \\
& +2 \eta \xi N_{\mu} \square Z^{\mu}-2 \eta(1-\xi) N_{\mu} N^{\mu}+(1-\eta) Z_{\mu \nu} \square Z^{\mu \nu}
\end{aligned}
$$

where $\eta, \rho$ and $\xi$ may take completely arbitrary values. We choose these parameters to streamline the present analysis. In particular, it is noteworthy that some of these parameters may be chosen to ensure the absence of four-fermion operators, which would otherwise complicate the calculation of observables.

Even after removing the four-fermi interactions in this way, there still remains a great deal of latitude in the form into which the effective theory may be cast. For example, even though our present lagrangian involves both vertex and self-energy corrections, we may use the equations of motion to transform all new-physics effects purely into self-energy corrections. In this case the techniques of ref. [21] may be used directly to bound the new physics. In the remainder of this paper we choose an alternative procedure. We instead recast our grown lagrangian into a form consisting of an 'STU'-sector (as in Eq. (18)), as well as a small set of higher-derivative vertex corrections (which, of course, do not contribute to low-energy observables). The parametrization of observables in terms of the six variables $S T U V W X$ nevertheless emerges at the end, as it must.

To obtain this final result requires specific choices for the operator transformations such as Eq. (36). We display the particular versions that we use in Table III. It is to be noted that an operator in the right column of this table is meant to be equivalent to the operator on the left to within $(i)$ total divergences, $(i i)$ terms which vanish with the SM equations of motion, and ( $i i i)$ terms which do not contribute to the well-measured physical processes we later consider. There are two varieties of terms of this last type. Firstly, some interactions have Feynman rules which are proportional to $q^{\mu} q^{\nu}$ and therefore do not contribute in the limit of massless fermions. Secondly, we omit terms such as fermion-fermion-photon-photon interactions, which cannot play a role at tree-level in the electroweak observables under consideration.

\section{3) Final Form of the 'Grown' Effective Lagrangian}

With the help of the transformations in Table III, $\mathcal{L}_{\text {grown }}$ can be recast as

$$
\mathcal{L}_{\text {grown }}^{\prime}=\mathcal{L}_{S T U}^{\prime}+\mathcal{L}_{\text {ver.corr. }}^{\prime}+\mathcal{L}_{\text {nonuniv. }}
$$




\begin{tabular}{cc}
\hline \hline Original Operator & Transformed Version of Operator \\
\hline$W_{\mu \nu}^{*} \square^{2} W^{\mu \nu}$ & $M_{W}^{4} W_{\mu \nu}^{*} W^{\mu \nu}+\left(J_{\mu} \square^{2} W^{* \mu}+h . c.\right)-M_{W}^{2}\left(J_{\mu} \square W^{* \mu}+h . c.\right)$ \\
$W_{\mu \nu}^{*} \square W^{\mu \nu}$ & $M_{W}^{2} W_{\mu \nu}^{*} W^{\mu \nu}+\left(J_{\mu} \square W^{* \mu}+h . c.\right)$ \\
$W_{\mu}^{*} J^{\mu}+W_{\mu} J^{* \mu}$ & $W_{\mu \nu}^{*} W^{\mu \nu}-2 M_{W}^{2} W_{\mu}^{*} W^{\mu}$ \\
$F_{\mu \nu} \square^{2} F^{\mu \nu}$ & $2 j_{\mu} \square^{2} A^{\mu}$ \\
$F_{\mu \nu} \square F^{\mu \nu}$ & $2 j_{\mu} \square A^{\mu}$ \\
$Z_{\mu \nu} \square^{2} F^{\mu \nu}$ & $2 j_{\mu} \square^{2} Z^{\mu}$ \\
$Z_{\mu \nu} \square F^{\mu \nu}$ & $2 j_{\mu} \square Z^{\mu}$ \\
$Z_{\mu \nu} \square^{2} Z^{\mu \nu}$ & $M_{Z}^{4} Z_{\mu \nu} Z^{\mu \nu}+2 N_{\mu} \square^{2} Z^{\mu}-2 M_{Z}^{2} N_{\mu} \square Z^{\mu}$ \\
$Z_{\mu \nu} \square Z^{\mu \nu}$ & $-M_{Z}^{2} Z_{\mu \nu} Z^{\mu \nu}+2 M_{Z}^{2} N_{\mu} \square Z^{\mu}$ \\
$N_{\mu} \square A^{\mu}$ & $j_{\mu} \square Z^{\mu}+(1 / 2) M_{Z}^{2} Z_{\mu \nu} F^{\mu \nu}$ \\
$N_{\mu} \square^{2} A^{\mu}$ & $j_{\mu} \square^{2} Z^{\mu}+M_{Z}^{2} j_{\mu} \square Z^{\mu}$ \\
\hline \hline
\end{tabular}

\section{TABLE III}

Operator Transformations Used to Simplify the Grown Lagrangian.

where $\mathcal{L}_{\text {nonuniv. }}$ contains the $m_{t}$-dependent effects, unchanged from eq. (12), and

$$
\begin{aligned}
\frac{1}{y} \mathcal{L}_{S T U}^{\prime}= & -\frac{A}{4} F_{\mu \nu} F^{\mu \nu}-\frac{B}{2} W_{\mu \nu}^{*} W^{\mu \nu}-\frac{C}{4} Z_{\mu \nu} Z^{\mu \nu} \\
& +\frac{G}{2} A_{\mu \nu} Z^{\mu \nu}+M_{W}^{2} w W_{\mu}^{*} W^{\mu}+\frac{M_{Z}^{2}}{2} z Z_{\mu} Z^{\mu}
\end{aligned}
$$

while

$$
\begin{aligned}
\frac{1}{y} \mathcal{L}_{\text {ver.corr. }}^{\prime}= & -\frac{1}{M_{Z}^{2}} M N_{\mu} \square Z^{\mu}+\frac{1}{M_{Z}^{4}} P N_{\mu} \square^{2} Z^{\mu} \\
& -\frac{1 s_{w}}{M_{Z}^{2} c_{w}}(M-N) j_{\mu} \square Z^{\mu}+\frac{1 s_{w}}{M_{Z}^{4} c_{w}}(P-Q) j_{\mu} \square^{2} Z^{\mu} \\
& -\frac{1}{M_{W}^{2}} H\left(J_{\mu} \square W_{\mu}^{*}+h . c .\right)+\frac{1}{M_{W}^{4}} K J_{\mu} \square^{2} W_{\mu}^{*} \\
& -\frac{1}{M_{Z}^{2}} D j_{\mu} \square A^{\mu}+\frac{1}{M_{Z}^{4}} E N_{\mu} \square^{2} A^{\mu}
\end{aligned}
$$

with $A, B$, etc. defined in Table IV.

The final version to which we have converted the grown lagrangian contains two categories of terms: mass and kinetic energy corrections for the gauge bosons (i.e. an $S T U$ 


\begin{tabular}{cc}
\hline \hline Parameter from Eqs. (38) and (39) & Definition \\
\hline$A$ & $-\alpha_{\gamma}$ \\
$B$ & $-\alpha_{W}-\beta_{W}-\gamma_{W}-2 p_{\mathrm{cc}}^{(0)}$ \\
$C$ & $-\alpha_{Z}-\beta_{Z}-\gamma_{Z}$ \\
$G$ & $+\alpha_{Z \gamma}-c_{w} p_{\mathrm{em}}^{(2)}$ \\
$w$ & $\omega_{W}-2 p_{\mathrm{cc}}^{(0)}$ \\
$z$ & $\omega_{Z}$ \\
$M$ & $\frac{\beta_{Z}}{2}+\frac{\gamma_{Z}}{2}+c_{w} p_{\mathrm{nc}}^{(2)}$ \\
$N$ & $\frac{\beta_{Z}}{2}+\frac{\gamma_{Z}}{2}-\frac{c_{w}}{s_{w}}\left(\beta_{Z \gamma}+c_{w} p_{\mathrm{em}}^{(2)}-c_{w} p_{\mathrm{em}}^{(4)}\right)$ \\
$P$ & $\frac{\gamma_{Z}}{2}+c_{w} p_{\mathrm{nc}}^{(4)}$ \\
$Q$ & $\frac{\gamma_{Z}}{2}-\frac{c_{w}}{s_{w}}\left(\gamma_{z \gamma}+c_{w} p_{\mathrm{em}}^{(4)}\right)$ \\
$\frac{\beta_{W}}{2}+\frac{\gamma_{W}}{2}+p_{\mathrm{cc}}^{(2)}$ \\
$\frac{\gamma_{W}}{2}+p_{\mathrm{cc}}^{(4)}$ \\
$s_{w} p_{\mathrm{em}}^{(2)}+\frac{\beta_{\gamma}}{2}$ \\
$D$ & $s_{w} p_{\mathrm{em}}^{(4)}+\frac{\gamma_{\gamma}}{2}$ \\
$E$ &
\end{tabular}

\section{TABLE IV}

Definitions of Parameters A,B,C, etc. Appearing in Final Form of 'Grown' Effective Lagrangian.

sector) and a limited number of dimension-six and dimension-eight vertex corrections. We have chosen this particular form for our grown lagrangian since the $S T U$ formalism can now be applied directly to the $S T U$ sector in the calculation of expressions for observables. As for the vertex corrections in $\mathcal{L}_{\text {grown }}^{\prime}$, they do not contribute to low-energy observables, and it is an easy matter to incorporate them in observables at other scales, especially since they have been consolidated into a reduced number of terms. In particular, as can be seen in Table III, we have decided to trade terms of type $N^{\mu} \square^{n} A_{\mu}$ for terms of type $j^{\mu} \square^{n} Z_{\mu}$; we find that this clarifies the calculation of neutral-current observables at the $Z$-pole by allowing us to consider only Z-exchange diagrams.

Importantly, this procedure hinges upon the fact that the grown currents can be expressed as linear combinations of total standard model currents, a property of all of the $T G V$-induced interactions except the $m_{t}$-dependent terms of $\mathcal{L}_{\text {nonuniv. }}$. 


\section{Expressions for Observables}

In this section, we show how the six parameters $(S, T, U, V, W$ and $X$ of ref. [21]) emerge in the present context. We do so by directly expressing the electroweak observables in terms of the coefficients in our effective lagrangian.

Since the calculation of the input observables $\alpha, G_{F}$ and $M_{Z}^{2}$ involves none of the higher-derivative vertex corrections, Eqs. (27) and (28) remain valid. Thus, using these results, we may immediately write the final total form of the various interaction sectors in terms of the canonically normalized fields. This entails simply adding the dimension-six and -eight vertex corrections to the pieces displayed in Eqs. (30) and (31):

$$
\begin{aligned}
\mathcal{L}_{\mathrm{nc}} & =-\frac{e}{c_{w} s_{w}}\left[\left(1+\frac{\alpha T}{2}\right) \bar{\Psi}_{f} \gamma_{\mu}\left[T_{3 f} \gamma_{L}-Q_{f}\left(s_{w}^{2}+\frac{\alpha}{4\left(c_{w}^{2}-s_{w}^{2}\right)} S-\frac{c_{w}^{2} s_{w}^{2} \alpha}{\left(c_{w}^{2}-s_{w}^{2}\right)} T\right)\right] \Psi_{f} Z^{\mu}\right. \\
& \left.-\bar{\Psi}_{f} \gamma_{\mu}\left[T_{3 f} M \gamma_{L}-Q_{f} s_{w}^{2} N\right] \Psi_{f} \frac{\square}{M_{z}^{2}} Z^{\mu}+\bar{\Psi}_{f} \gamma_{\mu}\left[T_{3 f} P \gamma_{L}-Q_{f} s_{w}^{2} Q\right] \Psi_{f} \frac{\square^{2}}{M_{z}^{4}} Z^{\mu}\right], \quad(40) \\
\mathcal{L}_{\mathrm{cc}} & =J_{\mu}\left(1-\frac{\alpha}{4\left(c_{w}^{2}-s_{w}^{2}\right)} S-\frac{s_{w}^{2} \alpha}{2\left(c_{w}^{2}-s_{w}^{2}\right)} T+\frac{\alpha}{8 s_{w}^{2}} U-H \frac{\square}{M_{W}^{2}}+K \frac{\square^{2}}{M_{W}^{4}}\right) W^{* \mu}+h . c ., \\
\mathcal{L}_{W} & =m_{z}^{2} c_{w}^{2}\left[1-\frac{\alpha S}{2\left(c_{w}^{2}-s_{w}^{2}\right)}+\frac{c_{w}^{2} \alpha T}{c_{w}^{2}-s_{w}^{2}}+\frac{\alpha U}{4 s_{w}^{2}}\right] W_{\mu}^{*} W^{\mu}, \\
\mathcal{L}_{\mathrm{em}} & =j_{\mu}\left(1-D \frac{\square}{M_{z}^{2}}+E \frac{\square^{2}}{M_{z}^{4}}\right) A^{\mu} .
\end{aligned}
$$

It is now a simple matter to use these interactions to derive expressions for various electroweak observables:

- Asymmetries: From Eq. (40), we deduce the Feynman rule

$$
\begin{aligned}
i \Lambda_{Z f f}^{\mu}\left(q^{2}\right)= & -i \frac{e}{c_{w} s_{w}}\left(1+\frac{\alpha T}{2}\right) \gamma^{\mu} \\
& {\left[T_{3 f}\left(1+M \frac{q^{2}}{M_{Z}^{2}}+P \frac{q^{4}}{M_{Z}^{4}}\right)\right.} \\
& \left.-Q_{f}\left(s_{w}^{2}+\frac{\alpha}{4\left(c_{w}^{2}-s_{w}^{2}\right)} S-\frac{c_{w}^{2} s_{w}^{2} \alpha}{\left(c_{w}^{2}-s_{w}^{2}\right)} T+s_{w}^{2}\left(N \frac{q^{2}}{M_{Z}^{2}}+Q \frac{q^{4}}{M_{Z}^{4}}\right)\right)\right]
\end{aligned}
$$

Forward-backward asymmetries and left-right asymmetries depend only on the 'effective' 
$\left(s_{w}\right)_{\text {eff }}\left(q^{2}\right)$, which can be read off from Eq. (44):

$$
\left(s_{w}^{2}\right)_{e f f}\left(q^{2}\right)=s_{w}^{2}+\frac{\alpha}{4\left(c_{w}^{2}-s_{w}^{2}\right)} S-\frac{c_{w}^{2} s_{w}^{2} \alpha}{\left(c_{w}^{2}-s_{w}^{2}\right)} T+s_{w}^{2}\left((N-M) \frac{q^{2}}{M_{Z}^{2}}+(Q-P) \frac{q^{4}}{M_{Z}^{2}}\right)
$$

In particular,

$$
\left(s_{w}^{2}\right)_{e f f}\left(M_{Z}^{2}\right)=s_{w}^{2}+\frac{\alpha}{4\left(c_{w}^{2}-s_{w}^{2}\right)} S-\frac{c_{w}^{2} s_{w}^{2} \alpha}{\left(c_{w}^{2}-s_{w}^{2}\right)} T+\alpha X
$$

where we define the new parameter $X$ according to

$$
\alpha X \equiv s_{w}^{2}(N+Q-M-P)
$$

For the specific case of asymmetries in the decay $Z \rightarrow b \bar{b}$, the effects of the nonuniversal $m_{t}$-dependent interactions must also be computed. Since these are not universal, they cannot be parameterized solely in terms of the variables $S$ through $X$. Their contribution to $Z \rightarrow b \bar{b}$ asymmetries can be included by replacing $X$ by $\hat{X}$, where

$$
\hat{X}=X+\frac{c_{w}^{2}}{8 \pi}\left|V_{t b}\right|^{2}\left(\frac{m_{t}^{2}}{M_{W}^{2}}\right)\left[3 \Delta g_{1 z}+\frac{1}{2 c_{w}^{2}} \Delta \kappa_{Z}\right] \log \left(\frac{\mu^{\prime 2}}{\mu^{2}}\right)
$$

- Z-Decay Widths: From Eq. (44) we obtain the partial width for $Z \rightarrow \bar{f} f$ :

$$
\Gamma_{Z f}=\frac{M_{Z}}{24 \pi} \frac{e^{2}}{c_{w}^{2} s_{w}^{2}}(1+\alpha T+\alpha V)\left[\left(T_{3 f}-Q_{f}\left(s_{w}^{2}\right)_{e f f}\left(M_{Z}^{2}\right)\right)^{2}+\left(Q_{f}\left(s_{w}^{2}\right)_{e f f}\left(M_{Z}^{2}\right)\right)^{2}\right]
$$

where we define the new parameter $V$ to be

$$
\alpha V \equiv 2(M+P)
$$

Again, the specific decay $Z \rightarrow b \bar{b}$ gets an additional contribution due to the $m_{t}$-dependence of the $T G V$-induced lagrangian. It may be incorporated by replacing $X$ by $\hat{X}$, as defined in eq. (48), in addition to making the replacement of $V$ by

$$
\hat{V}=V-\frac{c_{w}^{2}}{4 \pi s_{w}^{2}}\left|V_{t b}\right|^{2}\left(\frac{m_{t}^{2}}{M_{W}^{2}}\right)\left[3 \Delta g_{1 z}+\frac{1}{2 c_{w}^{2}} \Delta \kappa_{Z}\right] \log \left(\frac{\mu^{\prime 2}}{\mu^{2}}\right)
$$


- W-Decay Width: From Eq. (41) one obtains the Feynman rule

$$
i \Lambda_{\nu l W}^{\mu}\left(q^{2}\right)=-i \frac{e}{\sqrt{2} s_{w}} \gamma^{\mu}\left(1-\frac{\alpha}{4\left(c_{w}^{2}-s_{w}^{2}\right)} S-\frac{s_{w}^{2} \alpha}{2\left(c_{w}^{2}-s_{w}^{2}\right)} T+\frac{\alpha}{8 s_{w}^{2}} U+H \frac{q^{2}}{M_{W}^{2}}+K \frac{q^{4}}{M_{W}^{4}}\right) .
$$

Thus the partial width for $W \rightarrow l \nu_{l}$ is given by

$$
\Gamma_{W}=\frac{M_{W} e^{2}}{24 \pi 2 s_{w}^{2}}\left(1-\frac{\alpha}{2\left(c_{w}^{2}-s_{w}^{2}\right)} S-\frac{s_{w}^{2} \alpha}{\left(c_{w}^{2}-s_{w}^{2}\right)} T+\frac{\alpha}{4 s_{w}^{2}} U+\alpha W\right)
$$

where we define the new parameter

$$
\alpha W \equiv 2(H+K)
$$

- W Mass: The expression for $\mathrm{W}$ mass can be read off directly from Eq. (32):

$$
M_{W}^{2}=\left(M_{W}^{2}\right)_{S M}\left[1-\frac{\alpha S}{2\left(c_{w}^{2}-s_{w}^{2}\right)}+\frac{c_{w}^{2} \alpha T}{c_{w}^{2}-s_{w}^{2}}+\frac{\alpha U}{4 s_{w}^{2}}\right]
$$

- Low-Energy Observables: Let us now turn to the low-energy experiments such as deepinelastic scattering and atomic parity-violation. They are insensitive to the $q^{2}$ and $q^{4}$ pieces in the Eq. (40) which originate from the dimension-six and -eight vertex corrections in $\mathcal{L}_{\text {grown }}^{\prime}$. Therefore, these observables involve only $S, T$ and $U$, and the appropriate formulae can be taken directly from the usual $S T U$ treatment (see Appendix B in [11]).

A comprehensive list of expressions for the electroweak observables that we include in our analysis is given in Table $\mathrm{V}$. These expressions consist of a radiatively corrected standard model prediction plus a linear combination of the six parameters $S, T, U, V, W$ and $X . \Gamma_{Z}$ and $\Gamma_{b \bar{b}}$ are the total width of the $Z$ and its partial width into $b \bar{b}$, respectively; $A_{F B}(f)$ is the forward-backward asymmetry for $e^{+} e^{-} \rightarrow f \bar{f} ; A_{\text {pol }}(\tau)$, or $P_{\tau}$, is the polarization asymmetry defined by $A_{\text {pol }}(\tau)=\left(\sigma_{R}-\sigma_{L}\right) /\left(\sigma_{R}+\sigma_{L}\right)$, where $\sigma_{L, R}$ is the cross section for a correspondingly polarized $\tau$ lepton; $A_{e}\left(P_{\tau}\right)$ is the joint forward-backward/left-right asymmetry as normalized in ref. [25]; and $A_{L R}$ is the polarization asymmetry which has been measured by the SLD collaboration at SLC [26]. The low energy observables $g_{L}^{2}$ and $g_{R}^{2}$ are measured in deep inelastic $\nu N$ scattering, $g_{V}^{e}$ and $g_{A}^{e}$ are measured in $\nu e \rightarrow \nu e$ 
Expressions for Observables

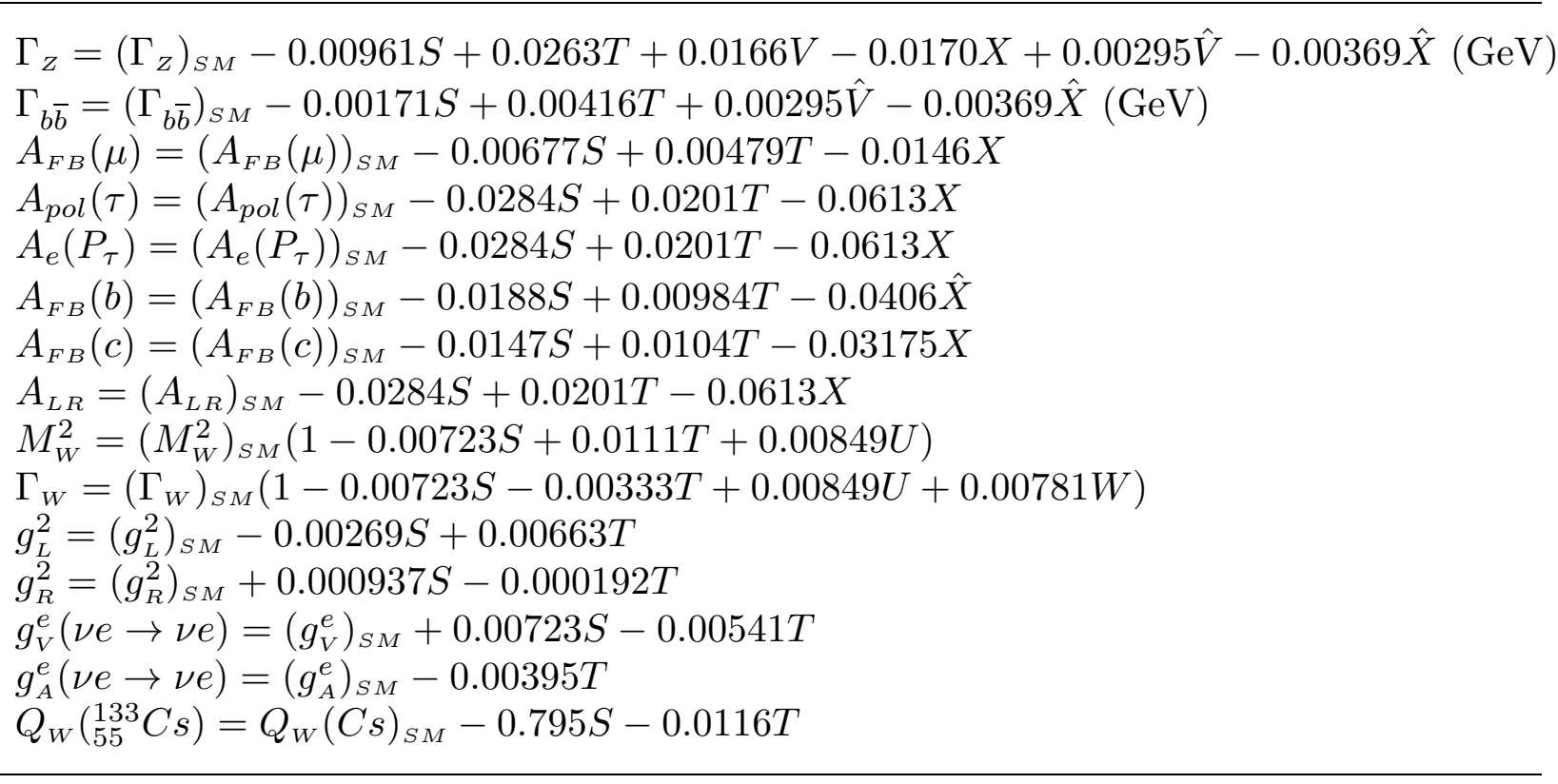

\section{TABLE V}

Summary of the dependence of electroweak observables on $S, T, U, V, W$ and $X$. In preparing this table we used the numerical values $\alpha\left(M_{Z}^{2}\right)=1 / 128$ and $s_{w}^{2}=0.23$.

scattering, and $Q_{W}(C s)$ is the weak charge measured in atomic parity violation in cesium. The expressions for the low energy observables are derived in refs. [11] and [24].

There are several features in Table $\mathrm{V}$ worth pointing out. First, only the two parameters $S$ and $T$ contribute to the observables for which $q^{2} \sim 0$. The parameter $U$ appears only in $M_{W}$ and $\Gamma_{W}$. Given the present uncertainty in $\Gamma_{W}$, the limit on $U$ comes from the $M_{W}$ measurement. The parameter $W$ is weakly bounded, as it contributes only to $\Gamma_{W}$ which is at present poorly measured. In addition to $S$ and $T$, observables on the $Z^{0}$ resonance are also sensitive to $V$ and $X$, which are expressly defined at $q^{2}=M_{Z}^{2}$. Observables that are not explicitly given in Table $\mathrm{V}$ can be obtained using the given expressions. In particular the parameter $R$ is defined as $R=\Gamma_{h a d} / \Gamma_{l \bar{l}}$, and $\sigma_{p}^{h}=12 \pi \Gamma_{e \bar{e}} \Gamma_{h a d} / M_{Z}^{2} \Gamma_{Z}^{2}$ is the hadronic cross section at the $Z$-pole. 


\section{Constraints on the Anomalous TGV Couplings}

We are now in a position to determine the phenomenological constraints on the anomalous TGV's. To do so, we must first consider how the well-measured couplings in the weak-scale effective lagrangian depend on the TGV parameters defined at the high scale, $\mu^{\prime}=M$.

In the previous section, we have derived (Table $\mathrm{V}$ ) expressions for observables in terms of $S$ through $X$. To obtain formulae for $S, T, U, V, W$ and $X$ in terms of the anomalous TGV's one substitutes the expressions in Tables I and II into those of Table IV, and then uses the definitions (29), (47), (50) and (54). The result of this substitution is displayed in Table VI, where we have taken $\mu^{\prime}=M=1 \mathrm{TeV}$ and $\mu=100 \mathrm{GeV}$.

\begin{tabular}{cc}
\hline \hline Parameter & One-Loop Result \\
\hline$S$ & $2.63 \Delta g_{1 z}-2.98 \Delta \kappa_{\gamma}+2.38 \Delta \kappa_{Z}+5.97 \lambda_{\gamma}-4.50 \lambda_{Z}$ \\
$T$ & $-1.82 \Delta g_{1 z}+0.550 \Delta \kappa_{\gamma}+5.83 \Delta \kappa_{Z}$ \\
$U$ & $2.42 \Delta g_{1 z}-0.908 \Delta \kappa_{\gamma}-1.91 \Delta \kappa_{Z}+2.04 \lambda_{\gamma}-2.04 \lambda_{z}$ \\
$V$ & $0.183 \Delta \kappa_{Z}$ \\
$W$ & $0.202 \Delta g_{1 z}$ \\
$X$ & $-0.0213 \Delta \kappa_{\gamma}-0.0611 \Delta \kappa_{Z}$ \\
$\hat{V}$ & $0.183 \Delta \kappa_{Z}-\left(3.68 \Delta g_{1 z}+0.797 \Delta \kappa_{Z}\right)\left(m_{t}^{2} / M_{W}^{2}\right)$ \\
$\hat{X}$ & $-0.0213 \Delta \kappa_{\gamma}-0.0611 \Delta \kappa_{Z}+\left(0.423 \Delta g_{1 z}+0.0916 \Delta \kappa_{Z}\right)\left(m_{t}^{2} / M_{W}^{2}\right)$ \\
\hline
\end{tabular}

\section{TABLE VI}

One-loop results for the induced parameters $S, T, U, V, W$ and $X$, defined at $\mu=100 \mathrm{GeV}$, in terms of the various TGV couplings defined at $M=1 \mathrm{TeV}$. We have used $\alpha\left(M_{Z}^{2}\right)=1 / 128$ and $s_{w}^{2}=0.23$.

To obtain constraints on the anomalous TGV's, we perform a global fit. The required expressions are obtained by substituting the results of Table VI into those of Table V. The TGV-dependence of the nonuniversal, $m_{t}$-dependent terms is given by eqs. (48) and (51).

The experimental values and standard model predictions of the observables used in our fit are given in Table VII. The standard model values have been calculated with $m_{t}=150$ $\mathrm{GeV}$ and $M_{H}=300 \mathrm{GeV}$. The LEP observables in Table VII were chosen as they are closest to what is actually measured and are relatively weakly correlated. In our analysis we include the combined LEP values for the correlations [27]. 


\begin{tabular}{ccc}
\hline \hline Quantity & Experimental Value & Standard Model Prediction \\
\hline$M_{Z}(\mathrm{GeV})$ & $91.187 \pm 0.007[28]$ & input \\
$\Gamma_{Z}(\mathrm{GeV})$ & $2.488 \pm 0.007[28]$ & $2.490[ \pm 0.006]$ \\
$R=\Gamma_{h a d} / \Gamma_{l \bar{l}}$ & $20.830 \pm 0.056[28]$ & $20.78[ \pm 0.07]$ \\
$\sigma_{p}^{h}(\mathrm{nb})$ & $41.45 \pm 0.17[28]$ & $41.42[ \pm 0.06]$ \\
$\Gamma_{b \bar{b}}(\mathrm{MeV})$ & $383 \pm 6[28]$ & $375.9[ \pm 1.3]$ \\
$A_{F B}(\mu)$ & $0.0165 \pm 0.0021[28]$ & 0.0141 \\
$A_{p o l}(\tau)$ & $0.142 \pm 0.017[28]$ & 0.137 \\
$A_{e}\left(P_{\tau}\right)$ & $0.130 \pm 0.025[28]$ & 0.137 \\
$A_{F B}(b)$ & $0.0984 \pm 0.0086[28]$ & 0.096 \\
$A_{F B}(c)$ & $0.090 \pm 0.019[28]$ & 0.068 \\
$A_{L R}$ & $0.100 \pm 0.044[26]$ & 0.137 \\
$M_{W}(\mathrm{GeV})$ & $79.91 \pm 0.39[29]$ & 80.18 \\
$M_{W} / M_{Z}$ & $0.8798 \pm 0.0028[30]$ & 2.082 \\
$\Gamma_{W}(\mathrm{GeV})$ & $2.12 \pm 0.11[31]$ & 0.3021 \\
\hline$g_{L}^{2}$ & $-71.04 \pm 1.58 \pm[0.88][32]$ & 0.0302 \\
$g_{R}^{2}$ & $0.3003 \pm 0.0039[25]$ & -0.506 \\
$g_{A}^{e}$ & $0.0323 \pm 0.0033[25]$ & -0.037 \\
$g_{V}^{e}$ & $-0.508 \pm 0.015[25]$ & \\
$Q_{W}(C s)$ & $-0.035 \pm 0.017[25]$ & \\
\hline \hline
\end{tabular}

\section{TABLE VII}

Experimental Values for Electroweak Observables Included in Global Fit. The $Z^{0}$ measurements are the preliminary 1992 LEP results taken from ref. [28]. The couplings extracted from neutrino scattering data are the current world averages taken from ref. [25]. The standard model values are for $m_{t}=$ $150 \mathrm{GeV}$ and $M_{H}=300 \mathrm{GeV}$. We have not shown theoretical errors in the standard model values due to uncertainties in the radiative corrections, $\Delta r$, and due to errors in $M_{Z}$, as they are in general overwhelmed by the experimental errors. The exception is the error due to uncertainty in $\alpha_{s}$, shown in square brackets. We include this error in quadrature in our fits. The error in square brackets for $Q_{W}(C s)$ reflects the theoretical uncertainty in the atomic wavefunctions [33] and is also included in quadrature with the experimental error.

As mentioned in the introduction, the same new physics responsible for the anomalous TGV's will typically also contribute directly to the various observables used in the fit. In 
our analysis we assume no cancellations between these 'direct' contributions and those due to the TGV's. Although this may seem like a very strong assumption, we will see that in any case the constraints obtained are rather weak.

We first consider the case in which only one of the TGV couplings, $\Delta \kappa_{V}, \lambda_{V}$ and $\Delta g_{1 z}$, is nonzero at the scale $M$. In this case strong bounds on this parameter may be obtained, since there is no possibility of cancellations. Constraining one parameter at a time we obtain the following values with $1 \sigma$ errors:

$$
\begin{aligned}
\Delta g_{1 z} & =-0.033 \pm 0.031 \\
\Delta \kappa_{\gamma} & =0.056 \pm 0.056 \\
\Delta \kappa_{z} & =-0.0019 \pm 0.044 \\
\lambda_{\gamma} & =-0.036 \pm 0.034 \\
\lambda_{z} & =0.049 \pm 0.045
\end{aligned}
$$

If taken at face value, these limits would imply that anomalous TGV's are too small to be seen at LEP200.

Of course, although the bounds obtained in this way are the tightest bounds that are possible, they are somewhat artificial. After all, real underlying physics would produce more than just a single TGV. If we fit for all five anomalous TGV's simultaneously, the constraints virtually disappear, due to the possibility of cancellations. Several authors have fitted $S T U$-corrected expressions for observables to electroweak data, and have concluded that the upper limit on these parameters is $O(0.1-1)$. It is clear that if one were to do fit a using the $S T U V W X$-corrected expressions displayed in Table $\mathrm{V}$, then the limits on the six parameters would be looser still. We find that at best, we can only conclude that the anomalous TGV couplings are less than $\mathcal{O}(1)$. TGV's of this size would, of course, be observable at LEP200.

The bounds given in eq. (56) are nevertheless interesting. These values can be interpreted as an indication of the sensitivity of the global fit of electroweak data to specific anomalous couplings. Once all of the couplings are allowed to vary simultaneously, no significant bound remains. This indicates that, in that part of the allowed region for which the TGV couplings are large, cancellations occur among the contribution of the various anomalous couplings to low-energy observables. Eq. (56) allows one to gain a feel for the size of cancellations that would be required to account for the low-energy data, should an anomalous TGV at the $10 \%$ level ever be discovered at LEP200.

Our results in this regard agree with those of ref. [17], who similarly obtain no significant bound for these couplings, subject to the somewhat stronger assumption that the 
effective theory be a linear realization of the electroweak gauge group. As is discussed in this reference, such a linear realization implies relationships among the various TGV parameters, and so would be expected to lead to tighter constraints than those obtained here. We here confirm this result within a more general phenomenological analysis, without theoretical biases.

\section{Conclusions}

We have computed the bounds that may be obtained for CP-preserving anomalous TGV's from current low-energy phenomenology. These bounds arise due to the influence of these interactions, through loops, on well-measured electroweak observables. We compute this influence using an effective-lagrangian description, in which TGV interactions are imagined to have been generated just below the scale for new physics, $\mu=M$, after all of the hitherto undiscovered heavy particles have been integrated out. Running this lagrangian, using the $\overline{\mathrm{MS}}$ renormalization scheme, down to the weak scale, $\mu \simeq 100 \mathrm{GeV}$, then generates a collection of secondary effective interactions. Unlike the TGV's, these new interactions contribute directly to low-energy observables, and so their couplings may be bounded by comparison to the data. We obtain limits on TGV's by requiring that the contributions to these couplings due to their RG mixing with the TGV's satisfy these experimental constraints. In so doing we are tacitly assuming that no cancellations arise between the induced values for these couplings, and their initial conditions at the newphysics scale, $\mu=M$.

An interesting feature of this calculation is that the weak-scale effective theory contains many higher-derivative interactions which contribute to both the gauge-boson propagation, as well as to the fermion-boson vertex corrections. We show how field redefinitions may be used to cast this collection of terms into a simplified form, rendering certain cancellations among the anomalous TGV's explicit at the outset. One approach involves putting all of the effects of new physics into the gauge-boson self-energies. Another approach (the one followed in the present article) involves recasting the effects of new physics into an $S T U$-sector plus a small set of higher-dimension fermion-boson vertex terms. In either case, since the resulting 'oblique' corrections necessarily involve higher powers of the momentumtransfer, $q^{2}$, they may not be parametrized purely in terms of the familiar variables $S, T$ and $U$. The three additional parameters, $V, W$ and $X$, of ref. [21] are also required.

The necessity for these additional parameters may come as something of a surprise, since we have assumed that the new physics scale, $M$, is large in comparison to the weak scale, $M_{Z}$. This would naively seem to justify the neglect of the higher-derivative oblique corrections, since these should be suppressed in their implications for low-energy observ- 
ables by factors of $M_{Z}^{2} / M^{2}$. The problem with this reasoning is that loop-induced effects to $S$ through $U$ are at most of order $(g / 4 \pi)^{2}$, and this is the same size as $M_{Z}^{2} / M^{2}$. As a result, TGV loop-induced corrections to the $S T U$ parameters are typically the same size as higher-derivative oblique corrections, and so these must be properly treated, as we have done.

We have found that the limits obtained in this way cannot in themselves rule out TGV's that are large enough to be detectable once LEP runs at the threshold for $W^{ \pm}$ pair production. Couplings of this size would have been ruled out if we had considered each TGV one at a time, with all of the others constrained to be zero. In this case the data would constrain the various TGV couplings to be $10 \%$ or less. This shows how (fairly mild) cancellations among the various TGV's would be required in low-energy observables should these TGV's be directly detected at LEP200. Since the underlying theory of new physics is unlikely to produce only one TGV at a time, this also shows how misleading can be the practice of working with TGV's one by one. A simultaneous fit of all of the TGV's to the data does not yield useful bounds.

An interesting feature of the expressions in Table VI is the occurence of some spectacular cancellations among anomalous TGV's. Although $S, T$ and $U$ contain all of the combinations of the anomalous TGV's, such is not the case for $V, W$ and $X$. In particular, the $\lambda_{V}$ couplings do not contribute to these parameters at all, $V$ depends only on $\Delta g_{1 z}$, and $X$ depends only on $\Delta \kappa_{V}$. This implies that each of the TGV couplings tends to contribute only to particular kinds of observables at $q^{2}=M_{Z}^{2}$ and $M_{W}^{2}$.

\section{Acknowledgments}

S.G. and D.L. gratefully acknowledge helpful conversations and communications with Paul Langacker. S.G. thanks Dean Karlen for useful exchanges. We also thank R. Sinha for interesting communications. This research was partially funded by funds from the

N.S.E.R.C. of Canada, les Fonds F.C.A.R. du Québec, and by the Deutsche Forschungsgemeinshaft. 


\section{Figure Captions}

- Figure (1): The Feynman graphs through which the anomalous three- and four-point gauge-boson vertices contribute to the gauge boson vacuum polarization. The blobs represent anomalous couplings, and all other interactions are standard.

- Figure (2): The Feynman graph through which the anomalous TGV's contribute to the gauge boson-fermion vertex corrections. The blob represents the anomalous TGV, and all other interactions are standard. 


\section{References}

[1] K. Hagiwara, R.D. Peccei, D. Zeppenfeld and K. Hikasa, Nucl. Phys. B282 (1987) 253.

[2] D. Zeppenfeld, Phys. Lett. 183B (1987) 380; D. Treille et al., Proceedings of the ECFA Workshop on LEP200 vol. 2, p4.14, A. Böhm, W. Hoogland, (eds) Aachen (1986) CERN 87-08; D. Dicus and K. Kallianpur, Phys. Rev. D32 (1985) 32.

[3] G. Kane, J. Vidal, C.P. Yuan, Phys. Rev. D39 (1989) 2617.

[4] A small sampling is: F. Herzog, Phys. Lett. 148B (1984) 355; M. Suzuki Phys. Lett. 153B (1985) 289; J.C. Wallet, Phys. Rev. D32 (1985) 813; A. Grau and J.A. Grifols, Phys. Lett. 154B (1985) 283; Phys. Lett. 166B (1986) 233; R. Alcorta, J.A. Grifols, and S. Peris, Mod. Phys. Lett. A2, (1987) 23; J.A. Grifols, S. Peris, and J. Solá, Phys. Lett. 197B (1987) 437; Int. J. Mod. Phys. A3 (1988) 225; P. Mery, S.E. Moubarik, M. Perrottet, and F.M. Renard, Zeit. Phys. C46 (1990) 229; G. Bélanger, F. Boudjema, and D. London, Phys. Rev. Lett. 65 (1990) 2943; S. Godfrey and H. König, Phys. Rev. D45 (1992) 3196; K.A. Peterson, Phys. Lett. 282B (1992) 207; T. G. Rizzo, Argonne report ANL-HEP-PR93-19 (1993; unpublished).

[5] M. Einhorn et.al., preprints UM-TH-93-12, UM-TH-92-25 (unpublished).

[6] S. Coleman, J. Wess and B. Zumino, Phys. Rev. 177 (1969) 2239; E.C. Callan, S. Coleman, J. Wess and B. Zumino, Phys. Rev. 177 (1969) 2247; J. Gasser and H. Leutwyler, Ann. Phys. (NY) 158 (1984) 142.

[7] A. Manohar and H. Georgi, Nucl. Phys. B234 (1984) 189; H.Georgi and L.Randall, Nucl. Phys. B276 (1986) 241; H. Georgi, Phys. Lett. 298B (1993) 187.

[8] A. de Rújula, M.B. Gavela, P. Hernandez and E. Massó, Nucl. Phys. B384 (1992) 3.

[9] M.E. Peskin and T. Takeuchi, Phys. Rev. Lett. 65 (1990) 964; D.C. Kennedy and P. Langacker, Phys. Rev. Lett. 65 (1990) 2967.

[10] W.J. Marciano and J.L. Rosner, Phys. Rev. Lett. 65 (1990) 2963

[11] M.E. Peskin and T. Takeuchi, Phys. Rev. D46 (1992) 381.

[12] J. Alitti et.al., The UA2 Collaboration, Phys. Lett. 277B (1992) 194. 
[13] J. Cortez, K. Hagiwara, F. Herzog, Nucl. Phys. B278 (1986) 26; M. Samuel et.al., Phys. Rev. Lett. 67 (1991) 9; Phys. Rev. D44 (1991) 2064; U. Baur and E.L. Berger, Phys. Rev. D41 (1990) 1476.

[14] U. Baur and D. Zeppenfeld, Nucl. Phys. B308 (1988) 127; D. Zeppenfeld and S. Willenbrock, Phys. Rev. D37 (1988) 1775; U. Baur and D. Zeppenfeld, Phys. Lett. 201B (1988) 383; Nucl. Phys. B308 (1988) 127; F. Pastore and M. Pepe, Proceedings of the Large Hadron Collider Workshop, Aachen, Vol II, p. 106, 1990, CERN 90-10.

[15] C.P. Burgess and D. London, preprint McGill-92/05, UdeM-LPN-TH-84 (to appear in Phys. Rev. D).

[16] K. Hagiwara, et al. MAD/PH 690 (1992).

[17] K. Hagiwara, S. Ishihara, R. Szalapski and D. Zeppenfeld, preprint MAD/PH/737, UT-635, KEK-TH-356, KEK preprint 92-214 (unpublished)

[18] P. Hernández and F.J. Vegas, Phys. Lett. 307B (1993) 116.

[19] D. Choudhury, P. Roy and R. Sinha, preprint TIFR-TH/93-08 (unpublished).

[20] J.M. Cornwall, D.N. Levin and G. Tiktopoulos, Phys. Rev. D10 (1974) 1145; M.S. Chanowitz, M. Golden and H. Georgi, Phys. Rev. D36 (1987) 1490.

[21] I. Maksymyk, C.P. Burgess and D. London, preprint McGill-93/13, UdeM-LPN-TH93-151, hepph-9306267 (unpublished).

[22] This observation goes back more than ten years to early applications of effective lagrangians to gravity and string theory, see e.g., M. Green, J. Schwarz and E. Witten, Superstring Theory (Cambridge University Press, 1987).

More recent applications in the SM literature may be found in C.P. Burgess and J.A. Robinson, in BNL Summer Study on CP Violation, S. Dawson and A. Soni editors, (World Scientific, Singapore, 1991); H. Georgi, Nucl. Phys. B361 (1991) 339.

[23] K.J.F. Gaemers and G.J. Gounaris, Zeit. Phys. C1 (1979) 259.

[24] C.P. Burgess, S. Godfrey, H. König, D. London and I. Maksymyk, preprint McGill93/12, UdeM-LPN-TH-93-155, OCIP/C-93-6 (unpublished).

[25] P. Langacker, to appear in the Proceedings of 30 Years of Neutral Currents, Santa 
Monica, February 1993.

[26] K. Abe et al., Phys. Rev. Lett. 70 (1993) 2515.

[27] The LEP Collaborations: ALEPH, DELPHI, L3, and OPAL, Phys. Lett. 276B (1992) 247.

[28] C. DeClercqan, Proceedings of the Recontre de Moriond, Les Arcs France, March 1993; V. Innocente, ibid.

[29] R. Abe et al., Phys. Rev. Lett. 65 (1990) 2243.

[30] J. Alitti et al., Phys. Lett. 276B (1992) 354.

[31] Particle Data Group, Phys. Rev. D45 (1992) II.

[32] M.C. Noecker et al., Phys. Rev. Lett. 61 (1988) 310.

[33] S.A. Blundell, W.R. Johnson, and J. Sapirstein, Phys. Rev. Lett. 65 (1990) 1411; V.A. Dzuba et al., Phys. Lett. 141A (1989) 147. 


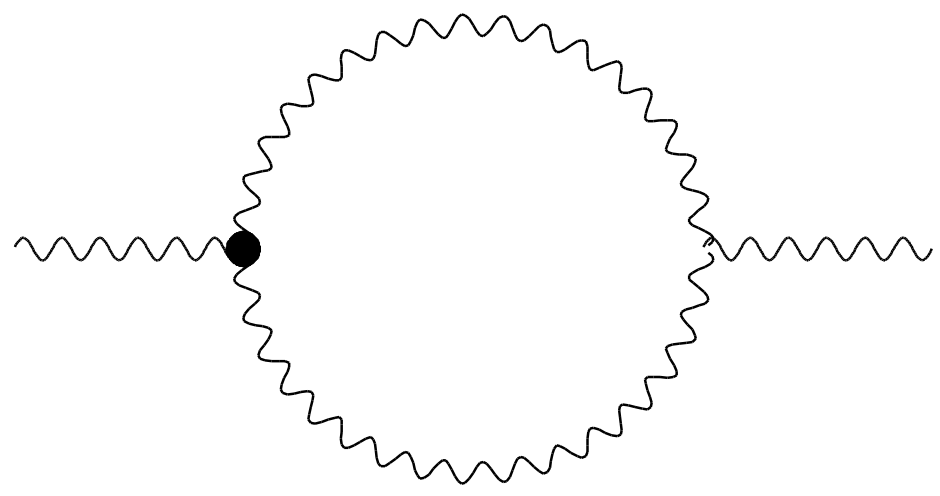




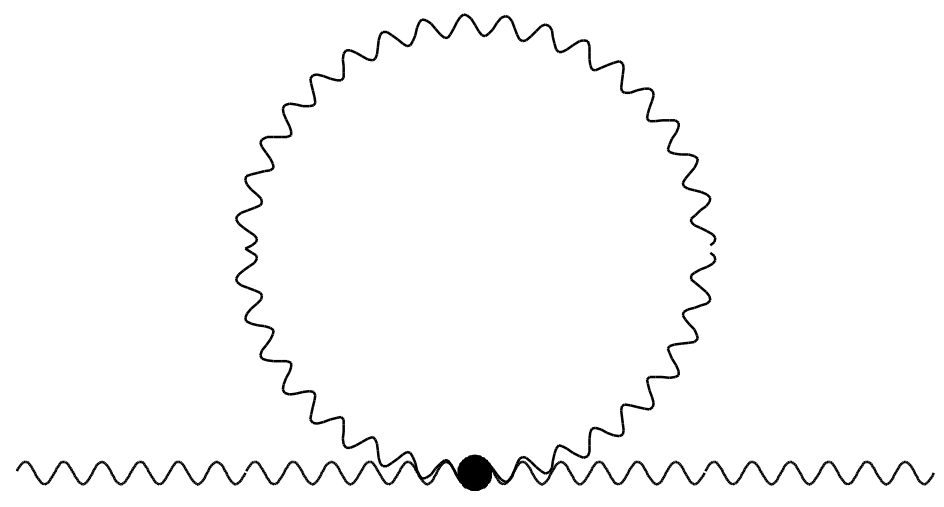




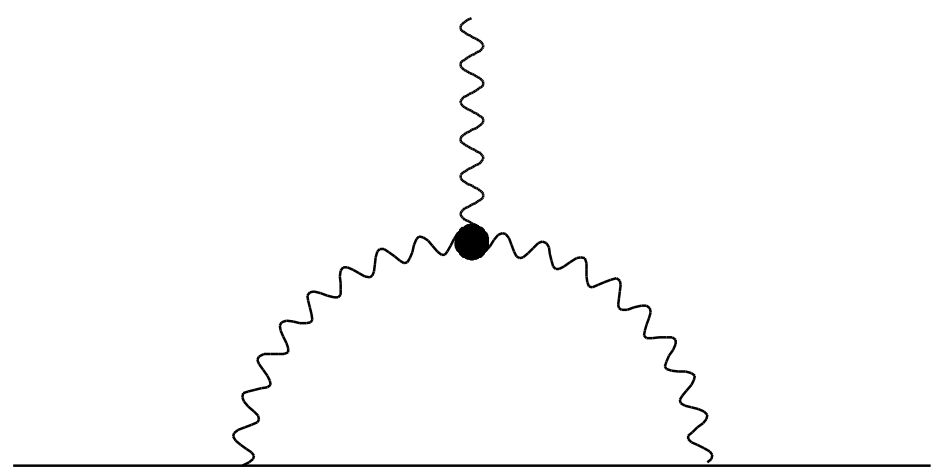

